\title{
Transcriptome Profiling of IL-17A Preactivated Mesenchymal Stem Cells: A Comparative Study to Unmodified and IFN- $\gamma$ Modified Mesenchymal Stem Cells
}

\author{
Kisha Nandini Sivanathan, ${ }^{1,2}$ Darling Rojas-Canales, ${ }^{1,2}$ Shane T. Grey, ${ }^{3}$ \\ Stan Gronthos, ${ }^{4,5}$ and Patrick T. Coates ${ }^{1,2,6}$ \\ ${ }^{1}$ School of Medicine, Faculty of Health Sciences, University of Adelaide, Adelaide, SA, Australia \\ ${ }^{2}$ Centre for Clinical and Experimental Transplantation, Royal Adelaide Hospital, Adelaide, SA, Australia \\ ${ }^{3}$ Transplantation Immunology Group, Garvan Institute of Medical Research, Sydney, NSW, Australia \\ ${ }^{4}$ South Australian Health and Medical Research Institute, Adelaide, SA, Australia \\ ${ }^{5}$ Mesenchymal Stem Cell Laboratory, School of Medicine, Faculty of Health Sciences, University of Adelaide, Adelaide, SA, Australia \\ ${ }^{6}$ Central Northern Adelaide Renal Transplantation Service, Royal Adelaide Hospital, Adelaide, SA, Australia
}

Correspondence should be addressed to Kisha Nandini Sivanathan; kisha.sivanathan@adelaide.edu.au

Received 21 September 2016; Accepted 20 December 2016; Published 15 February 2017

Academic Editor: Thomas Ichim

Copyright (C) 2017 Kisha Nandini Sivanathan et al. This is an open access article distributed under the Creative Commons Attribution License, which permits unrestricted use, distribution, and reproduction in any medium, provided the original work is properly cited.

\begin{abstract}
Human mesenchymal stem cells pretreatment with IL-17A (MSC-17) potently enhances T cell immunosuppression but not their immunogenicity, in addition to avidly promoting the induction of suppressive regulatory $\mathrm{T}$ cells. The aim of this study was to identify potential mechanisms by which human MSC-17 mediate their superior immunomodulatory function. Untreated-MSC (UT-MSC), IFN- $\gamma$ treated MSC (MSC- $\gamma$ ), and MSC-17 were assessed for their gene expression profile by microarray. Significantly regulated genes were identified for their biological functions (Database for Annotation, Visualisation and Integrated Discovery, DAVID). Microarray analyses identified 1278 differentially regulated genes between MSC- $\gamma$ and UT-MSC and 67 genes between MSC-17 and UT-MSC. MSC- $\gamma$ were enriched for genes involved in immune response, antigen processing and presentation, humoral response, and complement activation, consistent with increased MSC- $\gamma$ immunogenicity. MSC-17 genes were associated with chemotaxis response, which may be involved in T cell recruitment for MSC-17 immunosuppression. MMP1, MMP13, and CXCL6 were highly and specifically expressed in MSC-17, which was further validated by real-time PCR. Thus, MMPs and chemokines may play a key role in mediating MSC-17 superior immunomodulatory function. MSC-17 represent a potential cellular therapy to suppress immunological $\mathrm{T}$ cell responses mediated by expression of an array of immunoregulatory molecules.
\end{abstract}

\section{Introduction}

Human bone marrow derived mesenchymal stem cells (MSC) pretreated with interleukin-17A (IL-17A) represent a novel immunomodulatory strategy and an alternative to interferongamma (IFN- $\gamma$ ) treatment of MSC in enhancing MSC immunosuppression of $\mathrm{T}$ cells [1]. We have previously demonstrated that human MSC-17 potently suppresses human T cell proliferation and activation. In cocultures of MSC with purified human $\mathrm{CD} 4^{+} \mathrm{CD} 25^{-} \mathrm{T}$ cells, MSC-17 induced high numbers of functionally suppressive iTregs [1]. Whilst MSC17 appeared to be superior modulators of T cells, mechanisms exclusive to MSC-17 mediated immunomodulation warrant further investigation.

IL-17A is a member of the family of IL-17 cytokines secreted predominantly by the T helper 17 (Th17) subset of $\mathrm{CD} 4^{+} \mathrm{T}$ cells. IL-17A is a potent proinflammatory mediator and is involved in the pathogenesis of autoimmune diseases, allergic responses, and other immune cell mediated diseases including allograft rejection, sepsis, and graft versus 
host disease (GvHD) [2, 3]. Apart from the pathogenic roles of IL-17A, this cytokine is important for host defense response against fungal and bacterial infections $[3,4]$. The IL-17A homodimer signals through the IL-17RA and IL17RC dimeric receptor complex, where binding of IL-17A homodimer to the IL-17RA/RC complex recruits the key cytosolic adaptor molecule Actl (NF-kappa B-activating protein), that is known to be the master mediator of downstream IL-17 signaling $[3,5]$. Actl binds to the IL17RA/RC complex via its SEFIR (SEF/IL-17R) domains and this complex then recruits TRAF6 (TNF receptor-associated factor 6), leading to the activation of several downstream signaling pathways including the MAPKs-AP-1 (mitogenactivated protein kinases, MAPKs; activator protein-1, AP1), C/EBPs (CCAAT/enhancer-binding proteins) and $\mathrm{NF} \kappa \mathrm{B}$ (nuclear factor kappa B). Activation of these signaling cascades induces the gene expression of antimicrobial peptides, chemokines, MMPs, and proinflammatory cytokines as shown in other cell types such as endothelial cells, epithelial cells, and fibroblasts [3, 4]. IL-17A has emerged to be a growth factor for MSC by activating the Akt-Erk-MEK-p38 transduction molecules involved in MAPK signaling cascades [68]. Published work from our laboratory, demonstrated for the first time that IL-17A also enhances the immunomodulatory capacity of human MSC [1].

IFN- $\gamma$ is produced predominantly by $\mathrm{CD}^{+} \mathrm{T}$ cells and $\mathrm{NK}$ cells and at lower levels by $\mathrm{CD} 4^{+} \mathrm{T}$ cells [9]. IFN- $\gamma$ binds to a heterodimeric cell surface receptor complex consisting of the interferon-gamma receptor 1 (IFNGR1) and IFGR2, activating the classical JAK-STAT (signal transducer and activator of transcription) signaling pathways [10]. Activation of this pathway regulates several downstream cascades and induces expression of many genes, thereby contributing to the diverse biological effects of IFN- $\gamma$ in different cell types [1012]. IFN- $\gamma$ activates macrophages to induce antitumor [13] and antimicrobial activities [14]. It is also well established that IFN- $\gamma$ induces antigen processing and presentation pathways in different cell types for MHC antigen presentation to $\mathrm{T}$ cells $[9,15-17]$. In B cells, IFN- $\gamma$ regulates immunoglobulin production and class switching $[16,18]$. IFN- $\gamma$ also attracts leukocytes and favours the growth, differentiation, and maturation of many cells types $[11,16]$. IFN- $\gamma$ is classically known as a cytokine that favours Th1 cell development $[16,19]$. In an allotransplantation setting, IFN- $\gamma$ promotes antigenspecific Th1 differentiation that drives cell mediated allograft rejection [20]. Together, these findings suggest the potent proinflammatory role of IFN- $\gamma$.

The role of IFN- $\gamma$ in MSC immunomodulation, reparative properties, and homing potential has been extensively reviewed as previously published [21]. IFN- $\gamma$ treated MSC (MSC- $\gamma$ ) have enhanced immunomodulatory properties but are potentially immunogenic when administered in allogeneic or third-party hosts [1]. In this study, microarray and bioinformatics approaches were used to further identify novel candidate molecules expressed by MSC- $\gamma$ and MSC17 that enhance the immunomodulatory properties of MSC. Genes and biological processes that may contribute to MSC- $\gamma$ immunogenicity in allogeneic or third-party hosts were also explored.

\section{Materials and Methods}

2.1. MSC Culture and Characterisation. Human bone marrow aspirates were obtained from the posterior iliac crest of normal adults volunteers (subjects with informed consent; age 20-35 yr) according to guidelines approved by the Human Ethics Committee of the Royal Adelaide Hospital, Australia (Protocol 940911a). Bone marrow derived MSC cultures were established and maintained as previously described $[22,23]$. Cryopreserved MSC were cultured to log-phase and used at passage 6 in experiments. The immunophenotype of culture expanded MSC and their ability to differentiate into adipocytes, osteocytes, or chondrocytes have been confirmed and published [1].

2.2. Cytokine Treatment of MSC. MSC were seeded in tissue culture flasks at a density of 4000 cells $/ \mathrm{cm}^{2}$ and were allowed to adhere overnight. Fresh MSC media containing either no cytokines or recombinant human cytokines, $500 \mathrm{U} / \mathrm{ml}$ IFN$\gamma$ (eBioscience) or $50 \mathrm{ng} / \mathrm{ml}$ IL-17A (Peprotech), were added to the MSC cultures to derive UT-MSC, MSC- $\gamma$, or MSC17, respectively. At day 5, cytokines were washed out with Hank's Balanced Salt Solution (HBSS, Sigma) and modified MSC were used for microarray gene expression profiling and analysis.

2.3. Human MSC RNA Isolation. MSC were harvested using $0.25 \%$ trypsin/EDTA (Sigma) for $4 \mathrm{~min}, 37^{\circ} \mathrm{C}$, and rinsed with $5 \% \mathrm{FBS} / \mathrm{HBSS}$ and RNA was extracted according to the protocol established by the Adelaide Microarray Centre (http://www.microarray.adelaide.edu.au/protocols/). Briefly, total RNA was extracted by dissolving the cell pellet in $500 \mu \mathrm{L}$ TRIzol reagent (Invitrogen) and $100 \mu \mathrm{L}$ chloroform was added to the mixture. The mixture was kept on ice for $15 \mathrm{~min}$ followed by centrifugation at $6500 \times \mathrm{g}$ for $30 \mathrm{~min}$, $4^{\circ} \mathrm{C}$. The upper aqueous phase was retained and mixed with an equal volume of $70 \%$ ethanol in diethylpyrocarbonate $\mathrm{H}_{2} \mathrm{O}$. Total RNA was further purified using the RNeasy mini kit (Qiagen) with the following modification: DNA was digested using the DNase I from the RNase-free DNase set (Qiagen). The quantity of total RNA was measured using NanoDrop 1000 (Thermo Scientific). Samples were adjusted to a concentration of $100 \mathrm{ng} / \mu \mathrm{L}$ for microarray and were sent to the Adelaide Microarray Centre, University of Adelaide, for microarray gene expression profiling. The RNA sample was determined using the Agilent RNA Bioanalyzer. Only RNA samples with RNA integrity number (RIN) of $\geq 8$ were used for microarray analysis.

2.4. Microarray Analysis. RNA extracted from human MSC samples were analysed using the Affymetrix Human Gene 2.0 ST Array (Affymetrix Inc., High Wycombe, UK) for gene expression profiling. Microarray gene expression profiling was performed on UT-MSC, MSC- $\gamma$ and MSC-17 from 3 human MSC donor biological replicates (passage 6). Microarray experiments were conducted by the Adelaide Microarray Centre, University of Adelaide. 
2.5. Microarray Quality Control and Gene Expression Analysis. Probe cell intensity (CEL) files were obtained from the Adelaide Microarray Centre. The Expression Console Software (Affymetrix) was used for data quality control, normalization, and differential gene level analysis. CEL files of each array showed no major issues or damage with the images. No outlier samples were identified based on the configurable QA/QC metrics. The RMA (robust multiarray analysis) algorithm was used to perform background subtraction, normalization, and summarization of probe sets. CHP files were generated from the Expression Console Software for further Principal Component Analysis (PCA) and gene level summarization using the Transcriptome Analysis Console (TAC) software (Affymetrix). After normalization, UT-MSC, MSC- $\gamma$, and MSC-17 from 3 donor samples of each treatment group were averaged and an unpaired one-way ANOVA was performed with significantly regulated genes identified by $p<0.05$ and fold changes $<-2$ and $>2$. Gene lists for comparison of MSC-17 versus UT-MSC, MSC- $\gamma$ versus UT-MSC, and MSC-17 versus MSC- $\gamma$ were generated for subsequent bioinformatics analysis.

2.6. Functional Enrichment Analysis by DAVID. Gene lists for comparison of MSC-17 versus UT-MSC, MSC- $\gamma$ versus UT-MSC, and MSC-17 versus MSC- $\gamma$ were analysed for their biological functions using the Database for Annotation, Visualisation and Integrated Discovery (DAVID; https://david.ncifcrf.gov/). The gene list was uploaded using the official gene symbol onto DAVID for functional annotation clustering analysis with medium classification stringency, enrichment scores $>1.5$, and $p<0.05$ [24]. Functional annotation clustering analysis based on DAVID's default settings was performed. The gene sets were also subcategorised based on functional annotation of interest such as biological process (GOTERM_BP_FAT), molecular function (GOTERM_MF_FAT), and cellular component (GOTERM_CC_FAT).

2.7. Real-Time PCR Gene Validation. Genes of interest identified by microarray were validated by real-time PCR (RT-PCR) as previously described [1]. Gene specific human Taqman ${ }^{\circledR}$ primers MMP1 (Hs00899659_m1), MMP13 (Hs00233992_ml), CCL2 (Hs00234140_m1), CCL8 (Hs04187715_m1), CXCL6 (Hs00605742_m1), C3 (Hs00163811_ml), CH25H (Hs02379634_s1), and LBP (Hs01084621_ml) (Applied Biosystems) were used for gene expression analysis. Samples were run in triplicate and data were presented and normalized to the housekeeping gene hypoxanthine phosphoribosyltransferase-1 (HPRT1) (Hs99999909_ml). Mean normalized expression was calculated using the Qgene Module software as previously described [25].

\section{Results}

3.1. Transcriptome Profiling of UT-MSC, MSC- $\gamma$, and MSC17. The transcriptome differences between UT-MSC, MSC- $\gamma$, and MSC-17 from 3 different human MSC donors were compared in this study. Principal Component Analysis (PCA) was

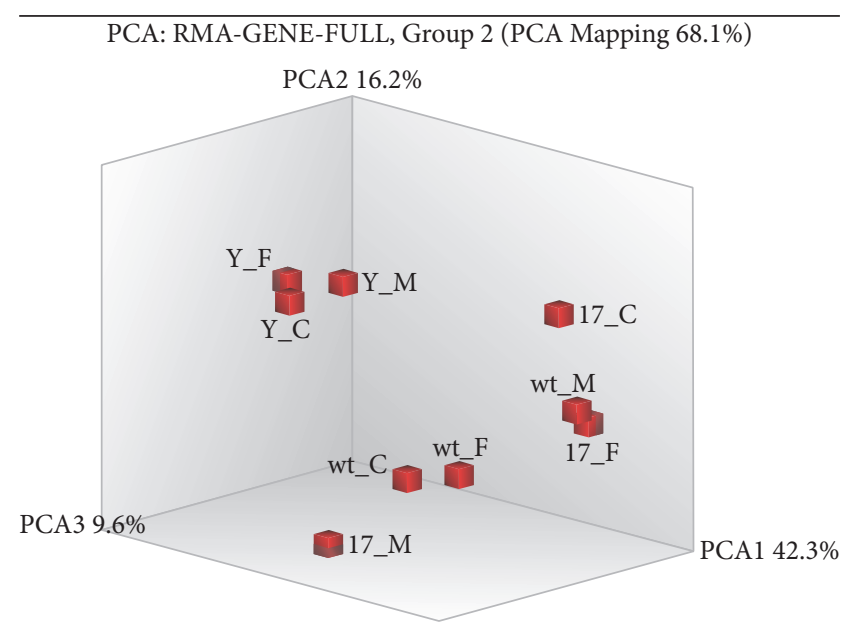

FIgure 1: Principal Component Analysis (PCA) of UT-MSC, MSC$\gamma$, and MSC-17. This 3-dimensional PCA graph identifies a new set of variables (PCA1, PCA2, and PCA3) that account for majority of the variability among the samples. PCA1 captures as much variability in the data as possible, PCA2 captures as much variability of the remaining variability not accounted by PCA1, and PCA3 captures as much of the remaining variability not accounted by PCA2. The symbols indicate IL-17A treated MSC, 17_; IFN- $\gamma$ treated MSC (Y_); and untreated-MSC (wt_). The 3 different MSC donors are indicated by $\mathrm{C}, \mathrm{M}$, and $\mathrm{F}$.

performed to visualise variances between the 3 donors and treatment groups. PCA analysis revealed that the 3 donor replicates of MSC- $\gamma$ "clustered" together. The gene expression pattern in the MSC- $\gamma$ groups were clearly distinct from UTMSC and MSC-17 (Figure 1). Microarray analysis revealed that 1278 genes (902 upregulated; 376 downregulated) were differentially regulated between MSC- $\gamma$ and UT-MSC. The top 30 upregulated and downregulated genes in the MSC- $\gamma$ were shown in Table 1.

There were however donor variances that exist between MSC-17 and UT-MSC. Among the 3 MSC donor samples evaluated, 2 MSC donors (i.e., donor C and F) "clustered" together and were distinct from UT-MSC (Figure 1). It should also be noted that in donor C and F MSC-17 "clusters," there was less variability in the gene expression profile in MSC-17 versus UT-MSC compared to the MSC- $\gamma$ versus UT-MSC groups. Donor M on the contrary had a different gene expression pattern in both UT-MSC and MSC-17. This clustering analysis in general supports a lesser degree of change in the gene expression profile of MSC with IL-17A than IFN $-\gamma$. Based on these 3 MSC donors, microarray analysis identified that only 67 genes (39 upregulated; 28 downregulated) were differentially regulated between MSC17 and UT-MSC (Table 2).

The gene expression profile of MSC-17 versus MSC- $\gamma$ was also evaluated. The clustering of the $3 \mathrm{MSC}$ donors in the MSC-17 and MSC- $\gamma$ comparison groups was more distinct (Figure 1) when compared to MSC-17 and UT-MSC. Microarray analysis revealed that 1806 genes (391 upregulated; 1415 downregulated) were differentially regulated between MSC17 and MSC- $\gamma$. The top 30 upregulated and downregulated 
TABLE 1: Top 30 differentially expressed genes: MSC- $\gamma$ versus UT-MSC.

\begin{tabular}{|c|c|c|c|c|}
\hline Gene symbol & Gene name & mRNA Accession & Fold change & $p$ value \\
\hline \multicolumn{5}{|c|}{ Upregulated genes } \\
\hline HLA-DRA & Major histocompatibility complex, class II, DR alpha & NM_019111 & 387.78 & 0.00049 \\
\hline GBP4 & Guanylate binding protein 4 & NM_052941 & 199.41 & 0.00002 \\
\hline IDO1 & Indoleamine 2,3-dioxygenase 1 & NM_002164 & 96.72 & 0.00003 \\
\hline HLA-DRB & Major histocompatibility complex, class II, DR beta & ENST00000307137 & 89.67 & 0.00435 \\
\hline GBP5 & Guanylate binding protein 5 & NM_052942 & 88.07 & 0.00003 \\
\hline CXCL9 & Chemokine (C-X-C motif) ligand 9 & NM_002416 & 83.60 & 0.00002 \\
\hline GBP2 & Guanylate binding protein 2, interferon-inducible & ENST00000464839 & 77.00 & 0.00004 \\
\hline SECTM1 & Secreted and transmembrane $1 ;$ NULL & NM_003004 & 57.59 & 0.00002 \\
\hline HLA-DRB3 & Major histocompatibility complex, class II, DR beta 3 & ENST00000426847 & 51.65 & 0.00868 \\
\hline CIITA & $\begin{array}{l}\text { Class II, major histocompatibility complex, } \\
\text { transactivator }\end{array}$ & NM_00024 & 38.84 & 0.00003 \\
\hline GBP1 & Guanylate binding protein 1 , interferon-inducible & NM_002053 & 29.09 & 0.00001 \\
\hline RP11-44K6.2 & NULL & ENST00000520185 & 26.39 & 0.00060 \\
\hline $\mathrm{GCH} 1$ & GTP cyclohydrolase 1 & NM_000161 & 24.93 & 0.00022 \\
\hline USP30-AS1 & USP30 antisense RNA 1 & ENST00000478808 & 24.75 & 0.00009 \\
\hline GBP2 & $\begin{array}{l}\text { Guanylate binding protein 2, interferon-inducible; } \\
\text { NULL }\end{array}$ & NM_004120 & 23.60 & 0.00001 \\
\hline HLA-DOA & $\begin{array}{l}\text { Major histocompatibility complex, class II, DO alpha; } \\
\text { NULL }\end{array}$ & NM_002119 & 22.90 & 0.00003 \\
\hline IFIT3 & $\begin{array}{l}\text { Interferon-induced protein with tetratricopeptide } \\
\text { repeats } 3\end{array}$ & NM_001031683 & 21.49 & 0.00013 \\
\hline FAM129A & Family with sequence similarity 129 , member A & NM_052966 & 20.89 & 0.00003 \\
\hline CTSS & Cathepsin S & NM_004079 & 20.10 & 0.00002 \\
\hline SLC7A11 & $\begin{array}{l}\text { Solute carrier family } 7 \text { (anionic amino acid transporter } \\
\text { light chain, xc- system), member } 11\end{array}$ & NM_014331 & 19.70 & 0.00009 \\
\hline IRF1 & Interferon regulatory factor 1 & NM_002198 & 19.55 & 0.00002 \\
\hline CD74 & $\begin{array}{l}\text { CD74 molecule, major histocompatibility complex, } \\
\text { class II invariant chain; NULL }\end{array}$ & NM_001025159 & 18.49 & 0.00060 \\
\hline ICAM1 & Intercellular adhesion molecule 1 & NM_000201 & 18.43 & 0.00003 \\
\hline $\mathrm{HCP} 5$ & HLA complex P5 (nonprotein coding); NULL & ENST00000457127 & 18.15 & 0.00032 \\
\hline LGALS17A & Charcot-Leyden crystal protein pseudogene & ENST00000412609 & 18.12 & 0.00038 \\
\hline PARP14 & Poly (ADP-ribose) polymerase family, member 14 & NM_017554; & 17.05 & 0.00014 \\
\hline RARRES3 & Retinoic acid receptor responder (tazarotene induced) 3 & NM_004585 & 17.00 & 0.00007 \\
\hline WARS & Tryptophanyl-tRNA synthetase; NULL & NM_004184 & 16.49 & 0.00002 \\
\hline IFIT2 & $\begin{array}{l}\text { Interferon-induced protein with tetratricopeptide } \\
\text { repeats } 2\end{array}$ & NM_001547 & 16.43 & 0.00051 \\
\hline TMEM140 & transmembrane protein 140 & NM_018295 & 16.08 & 0.00012 \\
\hline \multicolumn{5}{|c|}{ Downregulated genes } \\
\hline LRRC15 & Leucine rich repeat containing 15 & NM_001135057 & -19.91 & 0.0007 \\
\hline KIAA1199 & KIAA1199; NULL & NM_018689 & -13.26 & 0.0025 \\
\hline RNU5A-8P & RNA, U5A small nuclear 8, pseudogene & ENST00000364102 & -12.36 & 0.0061 \\
\hline COL10A1 & Collagen, type $\mathrm{X}$, alpha 1 & NM_000493; & -12.25 & 0.0031 \\
\hline COL3A1 & Collagen, type III, alpha 1; microRNA 3606 & NM_000090 & -11.88 & 0.0000 \\
\hline HIST1H2A & $\begin{array}{l}\text { Histone cluster 1, H2ai; histone cluster 1, H2ah; histone } \\
\text { cluster 1, H2ag; histone cluster 1, H2am; histone cluster } \\
\text { 1, H2al; histone cluster 1, H2ak; histone cluster 1, H3f }\end{array}$ & NM_003509 & -11.67 & 0.0012 \\
\hline SCD & Stearoyl-CoA desaturase (delta-9-desaturase) & NM_005063 & -9.75 & 0.0000 \\
\hline $\mathrm{U} 2$ & U2 spliceosomal RNA & ENST00000410792 & -9.41 & 0.0476 \\
\hline
\end{tabular}


TABLE 1: Continued.

\begin{tabular}{|c|c|c|c|c|}
\hline Gene symbol & Gene name & mRNA Accession & Fold change & $p$ value \\
\hline HIST1H3 & $\begin{array}{l}\text { Histone cluster 1, H3b; histone cluster 1, H3f; histone } \\
\text { cluster 1, H3h; histone cluster 1, H3j; histone cluster 1, } \\
\text { H3g; histone cluster 1, H3i; histone cluster 1, H3e; } \\
\text { histone cluster 1, H3c; histone cluster 1, H3d; histone } \\
\text { cluster 1, H3a }\end{array}$ & NM_003537 & -9.06 & 0.0053 \\
\hline HIST1H1B & Histone cluster $1, \mathrm{H} 1 \mathrm{~b}$ & NM_00532 & -8.53 & 0.0002 \\
\hline- & - & ENST00000408768 & -8.25 & 0.0001 \\
\hline KDELR3 & $\begin{array}{l}\text { KDEL (Lys-Asp-Glu-Leu) endoplasmic reticulum } \\
\text { protein retention receptor } 3\end{array}$ & NM_016657 & -8.22 & 0.0007 \\
\hline- & - & ВC091525 & -7.87 & 0.0007 \\
\hline SNORD114-11 & Small nucleolar RNA, C/D box 114-11 & NR_003204 & -7.33 & 0.0021 \\
\hline WISP1 & WNT1 inducible signaling pathway protein 1 & NM_003882 & -7.18 & 0.0000 \\
\hline U3 & Small nucleolar RNA U3 & ENST00000390893 & -7.14 & 0.0128 \\
\hline HIST1H2BM & Histone cluster $1, \mathrm{H} 2 \mathrm{bm}$ & NM_003521 & -6.92 & 0.0024 \\
\hline COL1A1 & Collagen, type I, alpha 1; NULL & NM_000088 & -6.84 & 0.0000 \\
\hline HIST1H3 & $\begin{array}{l}\text { histone cluster 1, H3g; histone cluster 1, H3f; histone } \\
\text { cluster 1, H3b; histone cluster 1, H3h; histone cluster 1, } \\
\text { H3j; histone cluster 1, H3i; histone cluster 1, H3e; } \\
\text { histone cluster 1, H3c; histone cluster 1, H3d; histone } \\
\text { cluster 1, H3a }\end{array}$ & NM_003534 & -6.68 & 0.0032 \\
\hline HIST1H3 & $\begin{array}{l}\text { Histone cluster 1, H3f; histone cluster 1, H3b; histone } \\
\text { cluster 1, H3h; histone cluster 1, H3j; histone cluster 1, } \\
\text { H3g; histone cluster 1, H3i; histone cluster 1, H3e; } \\
\text { histone cluster 1, H3c; histone cluster 1, H3d; histone } \\
\text { cluster 1, H3a }\end{array}$ & NM_021018 & -6.41 & 0.0058 \\
\hline AL732479.1 & - & ENST00000459197 & -6.38 & 0.0015 \\
\hline ADAM12 & ADAM metallopeptidase domain 12; NULL & NM_003474; & -6.13 & 0.0001 \\
\hline ENPP1 & Ectonucleotide pyrophosphatase/phosphodiesterase 1 & NM_006208 & -6.06 & 0.0002 \\
\hline NDNF & Neuron-derived neurotrophic factor & NM_024574 & -6.00 & 0.0100 \\
\hline DHCR7 & 7-Dehydrocholesterol reductase; NULL & NM_001360 & -5.88 & 0.0005 \\
\hline DHCR24 & 24-Dehydrocholesterol reductase & NM_014762 & -5.84 & 0.0001 \\
\hline RGS4 & Regulator of G-protein signaling 4; NULL & NM_001102445 & -5.78 & 0.0116 \\
\hline CRABP2 & Cellular retinoic acid binding protein 2 & NM_001878 & -5.76 & 0.0015 \\
\hline KIF20A & Kinesin family member 20A; NULL & NM_005733 & -5.60 & 0.0072 \\
\hline U1 & U1 spliceosomal RNA & - & -5.38 & 0.0069 \\
\hline
\end{tabular}

genes in the MSC-17 versus MSC- $\gamma$ comparison group were shown in Table 3. Volcano plots (Figure 2) and supervised hierarchical clustering of the differentially expressed genes (Figure 3) provided a global visualisation of genes regulated by IL-17 or IFN- $\gamma$ treatment of MSC compared to UT-MSC.

\subsection{MSC- $\gamma$ Enriched for Genes Associated with Increased} Immunogenicity. Upregulated and downregulated gene lists were submitted to DAVID for functional annotation clustering analysis to identify gene sets that were enriched in MSC- $\gamma$. There were 90 and 62 official gene symbols from the upregulated (see Table S1 in Supplementary Material available online at https://doi.org/10.1155/2017/1025820) and downregulated (Table S2) gene entry lists, respectively, that were unmapped by DAVID. These were mainly noncoding genes including microRNA (miRNA), long noncoding RNA
(lncRNA), and small nucleolar RNA (snoRNA). Gene ontology analysis by DAVID functional annotation clustering was performed on the upregulated and downregulated MSC- $\gamma$ versus UT-MSC gene lists to identify enriched gene sets for biological processes (Tables S3, S4), molecular functions (Tables S5, S6), and cellular components (Tables S7, S8).

Gene ontology analysis for biological processes of upregulated MSC- $\gamma$ genes (Table S3) uncovered highest enrichment of genes associated with antigen processing and presentation via MHC class I (annotation cluster 1, enrichment score 8.03). These genes were mainly HLA type genes and have roles in antigen presentation. Enriched genes in annotation cluster 1 also include aminopeptidases that hydrolyse antigenic peptides for MHC class I peptide binding and antigen presentation (e.g., endoplasmic reticulum aminopeptidase ERAP1 and ERAP2), peptide transporter 
TABLE 2: Differentially expressed genes (mapped by DAVID): MSC-17 versus UT-MSC.

\begin{tabular}{|c|c|c|c|c|}
\hline Gene symbol & Gene name & mRNA Accession & Fold change & $p$ value \\
\hline \multicolumn{5}{|c|}{ Upregulated genes } \\
\hline MMP13 & Matrix metallopeptidase 13 (collagenase 3) & NM_002427 & 15.60 & 0.0021 \\
\hline $\mathrm{C} 3$ & Complement component 3; NULL & NM_000064 & 11.56 & 0.0039 \\
\hline LBP & Lipopolysaccharide binding protein & NM_004139 & 5.35 & 0.0031 \\
\hline VMO1 & Vitelline membrane outer layer 1 homolog (chicken) & NM_182566 & 4.07 & 0.0022 \\
\hline $\mathrm{CH} 25 \mathrm{H}$ & Cholesterol 25-hydroxylase & NM_003956 & 3.99 & 0.0023 \\
\hline IL6 & Interleukin 6 (interferon, beta 2); NULL & NM_000600 & 3.44 & 0.0083 \\
\hline $\mathrm{ZC} 3 \mathrm{H} 12 \mathrm{~A}$ & Zinc finger CCCH-type containing 12A & NM_025079 & 3.09 & 0.0010 \\
\hline CCL2 & Chemokine (C-C motif) ligand 2 & NM_002982 & 3.08 & 0.0405 \\
\hline ZNF253 & Zinc finger protein 253 & NM_021047 & 2.82 & 0.0010 \\
\hline SAA1 & Serum amyloid A1 & NM_000331 & 2.72 & 0.0102 \\
\hline CXCL6 & Chemokine (C-X-C motif) ligand 6 & NM_002993 & 2.44 & 0.0014 \\
\hline MMP1 & Matrix metallopeptidase 1 (interstitial collagenase) & NM_002421 & 2.40 & 0.0356 \\
\hline NFKBIZ & $\begin{array}{l}\text { Nuclear factor of kappa light polypeptide gene enhancer in } \\
\text { B-cells inhibitor, zeta; NULL }\end{array}$ & NM_031419 & 2.36 & 0.0232 \\
\hline MIRLET7A2 & MicroRNA let-7a-2 & NR_029477 & 2.30 & 0.0031 \\
\hline RBMY2EP & $\begin{array}{l}\text { RNA binding motif protein, Y-linked, family 2, member E } \\
\text { pseudogene }\end{array}$ & ENST00000444169 & 2.27 & 0.0278 \\
\hline CCL8 & Chemokine (C-C motif) ligand 8 & NM_005623 & 2.20 & 0.0012 \\
\hline STC1 & Stanniocalcin 1 & NM_003155 & 2.20 & 0.0023 \\
\hline SFRP4 & Secreted frizzled-related protein 4 & NM_003014 & 2.19 & 0.0136 \\
\hline SLC22A3 & $\begin{array}{l}\text { Solute carrier family } 22 \text { (extraneuronal monoamine } \\
\text { transporter), member } 3\end{array}$ & NM_021977 & 2.15 & 0.0452 \\
\hline TTTY11 & Testis-specific transcript, Y-linked 11 (nonprotein coding) & NR_001548 & 2.15 & 0.0252 \\
\hline STEAP2 & STEAP family member 2 , metalloreductase; NULL & NM_001244944 & 2.12 & 0.0225 \\
\hline SCARNA18 & Small Cajal body-specific RNA 18 & NR_003139 & 2.06 & 0.0254 \\
\hline LOC100287834 & Uncharacterised LOC100287834 & NR_028349 & 2.06 & 0.0328 \\
\hline \multicolumn{5}{|c|}{ Downregulated genes } \\
\hline RPS24 & Ribosomal protein S24; NULL & NM_001142285 & -2.01 & 0.0209 \\
\hline LOC100133299 & GALI1870 & AY358688 & -2.03 & 0.0095 \\
\hline POU5F1 & POU class 5 homeobox 1 & ENST00000259915 & -2.04 & 0.0129 \\
\hline TMEM171 & Transmembrane protein 171 & NM_173490 & -2.05 & 0.0133 \\
\hline IGLJ2 & Immunoglobulin lambda joining 2 & ENST00000390322 & -2.07 & 0.0252 \\
\hline ITGA6 & Integrin, alpha 6; NULL & ENST00000264107 & -2.11 & 0.0109 \\
\hline RNU7-25P & $\begin{array}{l}\text { RNA, U7 small nuclear } 25 \text { pseudogene; RNA, U7 small } \\
\text { nuclear } 11 \text { pseudogene }\end{array}$ & ENST00000516544 & -2.16 & 0.0047 \\
\hline GTF2IRD2B & GTF2I repeat domain containing 2B & NM_001003795 & -2.20 & 0.0040 \\
\hline SERTAD4 & SERTA domain containing 4 & ENST00000367012 & -2.29 & 0.0470 \\
\hline TPTE & Transmembrane phosphatase with tensin homology & ENST00000415664 & -2.85 & 0.0128 \\
\hline
\end{tabular}

genes (e.g., transported associated with antigen processing, TAP2), and other genes involved in the antigen processing and presentation pathway (e.g., TAP binding protein, TAPBPL; $\beta_{2}$ microglobulin, B2M; CD74). Gene sets involved with antigen processing and presentation via MHC class II were also upregulated in the MSC $-\gamma$ groups (annotation cluster 4, enrichment score 4.45). In annotation cluster 2 (enrichment score 6.06) there were also enriched gene sets involved in immune response activation (innate, adaptive, and lymphocytes mediated immunity), humoral response (immunoglobulin mediated immune response, B cell mediated immunity, and humoral immune response mediated by circulating immunoglobulin), and complement pathways (classical and alternative) activation.

Apart from genes that are involved in increased MSC$\gamma$ immunogenicity, there were genes with regulatory roles upregulated in MSC- $\gamma$ (Table S3). For example, these gene sets were involved in the regulation of programmed cell 
TABLE 3: Top 30 differentially expressed genes: MSC-17 versus MSC- $\gamma$.

\begin{tabular}{|c|c|c|c|c|}
\hline Gene symbol & Gene name & mRNA Accession & Fold change & $p$ value \\
\hline \multicolumn{5}{|l|}{ Upregulated genes } \\
\hline MMP13 & Matrix metallopeptidase 13 (collagenase 3 ) & NM_002427 & 24.27 & 0.0009 \\
\hline HIST1H2AI & Histone cluster $1, \mathrm{H} 2 \mathrm{ai}$ & NM_003509 & 17.44 & 0.0005 \\
\hline U3 & Small nucleolar RNA U3 & ENST00000390893 & 13.96 & 0.0118 \\
\hline ZNF25 & Zinc finger protein 25 & ENSG00000175395 & 13.66 & 0.0008 \\
\hline LRRC15 & Leucine rich repeat containing 15 & NM_001135057 & 13.57 & 0.0005 \\
\hline HIST1H3G & Histone cluster $1, \mathrm{H} 3 \mathrm{~g}$ & NM_003534 & 12.68 & 0.0008 \\
\hline SNORD114-11 & Small nucleolar RNA, C/D box 114-11 & NR_003204 & 12.33 & 0.0386 \\
\hline HIST1H3B & Histone cluster $1, \mathrm{H} 3 \mathrm{~b}$ & NM_003537 & 11.20 & 0.0064 \\
\hline $\mathrm{U} 1$ & U1 spliceosomal RNA & NONHSAT054977 & 10.54 & 0.0017 \\
\hline HIST1H1B & Histone cluster $1, \mathrm{H} 1 \mathrm{~b}$ & NM_005322 & 10.28 & 0.0003 \\
\hline SCD & Stearoyl-CoA desaturase (delta-9-desaturase) & NM_005063 & 10.15 & 0.0008 \\
\hline KRT16P4 & Keratin 16 pseudogene 4 & ENST00000453883 & 9.11 & 0.0122 \\
\hline ADAM12 & ADAM metallopeptidase domain 12 & NM_001288973 & 8.96 & 0.0031 \\
\hline HIST1H2BM & Histone cluster 1, $\mathrm{H} 2 \mathrm{bm}$ & NM_003521 & 8.87 & 0.0125 \\
\hline HIST1H3F & Histone cluster $1, \mathrm{H} 3 \mathrm{f}$ & NM_021018 & 8.77 & 0.0081 \\
\hline ADAM12 & ADAM metallopeptidase domain 12 & NM_001288973 & 8.70 & 0.0121 \\
\hline KIAA1199 & KIAA1199; NULL & NM_018689 & 8.48 & 0.0045 \\
\hline COL10A1 & Collagen, type $\mathrm{X}$, alpha 1 & NM_000493 & 6.87 & 0.0136 \\
\hline DHCR7 & 7-Dehydrocholesterol reductase; NULL & NM_001360 & 6.51 & 0.0022 \\
\hline Р4HA3 & Prolyl 4-hydroxylase, alpha polypeptide III & NM_182904 & 6.49 & 0.0043 \\
\hline LBP & Lipopolysaccharide binding protein & NM_004139 & 6.11 & 0.0021 \\
\hline HAS1 & Hyaluronan synthase 1 & NM_001523 & 6.10 & 0.0038 \\
\hline COL1A1 & Collagen, type I, alpha 1; NULL & NM_000088 & 5.94 & 0.000002 \\
\hline NDNF & Neuron-derived neurotrophic factor & NM_024574 & 5.75 & 0.0147 \\
\hline ELN & Elastin; NULL & NM_000501 & 5.65 & 0.0014 \\
\hline WISP1 & WNT1 inducible signaling pathway protein 1 & NM_003882 & 5.61 & 0.0052 \\
\hline ADAM12 & ADAM metallopeptidase domain 12; NULL & NM_003474 & 5.56 & 0.0008 \\
\hline KDELR3 & $\begin{array}{l}\text { KDEL (Lys-Asp-Glu-Leu) endoplasmic reticulum } \\
\text { protein retention receptor } 3\end{array}$ & NM_016657 & 5.36 & 0.0085 \\
\hline HIST1H3I & Histone cluster $1, \mathrm{H} 3 \mathrm{i}$ & NM_003533 & 5.02 & 0.0014 \\
\hline CNN1 & Calponin 1, basic, smooth muscle & NM_001299 & 4.69 & 0.00001 \\
\hline \multicolumn{5}{|c|}{ Downregulated genes } \\
\hline HLA-DRA & Major histocompatibility complex, class II, DR alpha & NM_019111 & -553.64 & 0.0005 \\
\hline GBP4 & Guanylate binding protein 4 & NM_052941 & -244.27 & 0.0001 \\
\hline HLA-DRA & $\begin{array}{l}\text { Major histocompatibility complex, class II, DR alpha; } \\
\text { NULL }\end{array}$ & ENST00000442960 & -188.10 & 0.00005 \\
\hline CXCL9 & Chemokine (C-X-C motif) ligand 9 & NM_002416 & -96.44 & 0.00001 \\
\hline IDO1 & Indoleamine 2,3-dioxygenase 1 & NM_002164 & -76.98 & 0.00004 \\
\hline HLA-DRB3 & Major histocompatibility complex, class II, DR beta 3 & ENST00000307137 & -70.87 & 0.0024 \\
\hline GBP5 & Guanylate binding protein 5 & NM_052942 & -65.02 & 0.000004 \\
\hline SECTM1 & Secreted and transmembrane 1; NULL & NM_003004 & -50.74 & 0.0001 \\
\hline GBP2 & Guanylate binding protein 2, interferon-inducible & ENST00000464839 & -41.55 & 0.00001 \\
\hline HLA-DPA1 & $\begin{array}{l}\text { Major histocompatibility complex, class II, DP alpha } \\
1\end{array}$ & NM_001242524 & -40.79 & 0.0011 \\
\hline IFIT3 & $\begin{array}{l}\text { Interferon-induced protein with tetratricopeptide } \\
\text { repeats } 3\end{array}$ & NM_001031683 & -39.52 & 0.0011 \\
\hline CIITA & $\begin{array}{l}\text { Class II, major histocompatibility complex, } \\
\text { transactivator }\end{array}$ & NM_000246 & -37.92 & 0.000003 \\
\hline
\end{tabular}


TABle 3: Continued.

\begin{tabular}{|c|c|c|c|c|}
\hline Gene symbol & Gene name & mRNA Accession & Fold change & $p$ value \\
\hline GBP1 & Guanylate binding protein 1 , interferon-inducible & NM_002053 & -35.79 & 0.0002 \\
\hline PSAT1 & Phosphoserine aminotransferase 1 & NM_021154 & -31.20 & 0.0001 \\
\hline HLA-DPB1 & NULL & OTTHUMT00000310634 & -28.81 & 0.00002 \\
\hline GBP1P1 & $\begin{array}{l}\text { Guanylate binding protein 1, interferon-inducible } \\
\text { pseudogene } 1\end{array}$ & ENST00000513638 & -27.87 & 0.00001 \\
\hline GCH1 & GTP cyclohydrolase 1 & NM_000161 & -26.74 & 0.0002 \\
\hline PARP14 & Poly (ADP-ribose) polymerase family, member 14 & NM_017554 & -26.49 & 0.0008 \\
\hline IRF1 & Interferon regulatory factor 1 & NM_002198 & -24.92 & 0.000003 \\
\hline HLA-DOA & Major histocompatibility complex, class II, DO alpha & NM_002119 & -24.00 & 0.00001 \\
\hline RARRES3 & $\begin{array}{l}\text { Retinoic acid receptor responder (tazarotene } \\
\text { induced) } 3\end{array}$ & NM_004585 & -23.10 & 0.0001 \\
\hline LGALS17A & Charcot-Leyden crystal protein pseudogene & ENST00000412609 & -22.45 & 0.0003 \\
\hline HLA-DOA & Major histocompatibility complex, class II, DO alpha & NM_002119 & -21.85 & 0.00001 \\
\hline SLC7A11 & $\begin{array}{l}\text { Solute carrier family } 7 \text { (anionic amino acid } \\
\text { transporter light chain, xc- system), member } 11\end{array}$ & NM_014331 & -21.62 & 0.00004 \\
\hline USP30-AS1 & USP30 antisense RNA 1 & ENST00000478808 & -20.00 & 0.0001 \\
\hline HCP5 & HLA complex P5 (non-protein coding); NULL & ENST00000457127 & -19.90 & 0.0001 \\
\hline ICAM1 & Intercellular adhesion molecule 1 & NM_000201 & -19.63 & 0.0001 \\
\hline WARS & Tryptophanyl-tRNA synthetase; NULL & NM_004184 & -19.41 & 0.0001 \\
\hline APOL1 & Apolipoprotein L, 1; NULL & NM_003661 & -19.36 & 0.00002 \\
\hline ERVK-7 & $\begin{array}{l}\text { Endogenous retrovirus group } \mathrm{K} \text {, member } 7 \text {; novel } \\
\text { transcript }\end{array}$ & ENST00000522373 & -18.93 & 0.0267 \\
\hline HLA-DPB2 & $\begin{array}{l}\text { Major histocompatibility complex, class II, DP beta } 2 \\
\text { (pseudogene) }\end{array}$ & NR_001435 & -18.91 & 0.00009 \\
\hline
\end{tabular}

death, apoptosis, translation regulation, protein modification, transcription regulation and DNA binding activity, cell-cell communication, and signal transduction as well as the regulation of cytokine production. Moreover, genes upregulated in the MSC- $\gamma$ group were enriched for the TGF$\beta$ receptor signaling pathway (annotation cluster 19 , enrichment score 1.74, e.g., FMOD, CCL2, MAPK3K1, SMAD6, GDF15, and TGFB2). Other genes of interest upregulated in MSC- $\gamma$ include IL-6, toll-like receptor-3 (TLR3), TLR4, and indoleamine 2,3-dioxygenase (IDO), with the gene ontology term for positive regulation of defense response. There was also upregulation of the PD-L1 transcript in MSC- $\gamma$ compared to UT-MSC (3.46-fold, $p<0.0104$; data not shown), consistent with the observed increase in cell surface protein expression of PD-L1 following IFN- $\gamma$ pretreatment of MSC, as we have previously published [1]. Regulatory genes with nucleotide binding activity and transcription (corepressor, repressor, and cofactor) activity were also enriched and upregulated in MSC- $\gamma$ as identified by DAVID gene ontology analysis for molecular function (Table S5).

MSC $-\gamma$ have enhanced migratory potential to sites of inflammation [21]. Based on DAVID analysis for biological processes, we have identified gene sets in annotation cluster 10 (enrichment score 2.78) that were enriched for the gene ontology terms regulation of cell motion, cell migration, and locomotion (Table S3). These upregulated
MSC- $\gamma$ genes include chemokines (CXCL10, CXCL16), intracellular adhesion molecule-1 (ICAM1), IL-6, and VEGFA. The upregulation of chemotactic factors that may increase MSC- $\gamma$ homing potential was also identified when gene ontology analysis for molecular function was performed on DAVID. In annotation cluster 3 (enrichment score 3.10; Table S5), genes were enriched for chemokine receptor binding and chemokine activity. These chemokines include CCL13, CCL2, CXCL16, CXCL9, CCL8, CXCL11, and CXCL10.

Based on the downregulated MSC- $\gamma$ versus UT-MSC gene list, we identified that there were genes highly enriched for the gene ontology terms for biological processes involving extracellular matrix or structure organisation (annotation cluster 1, enrichment score 11.10; Table S4), consistent with our previous observation of changes in MSC- $\gamma$ morphology from fibroblastic-like appearance to a hypertrophic flattened irregular shape [1]. These were mainly collagen type genes (collagenases I, III, IV, V, XI, XII, and XIV). Interestingly, the downregulated gene sets also have enriched terms for biological processes involved in the cell division cycle (annotation cluster 2, enrichment score 8.80; Table S4) These downregulated genes were essential for $M$ phase, nuclear division, mitosis, and cell division. Genes essential for regulation of cell-cycle division were also downregulated in MSC- $\gamma$ (annotation cluster 7, enrichment score 2.03), in 


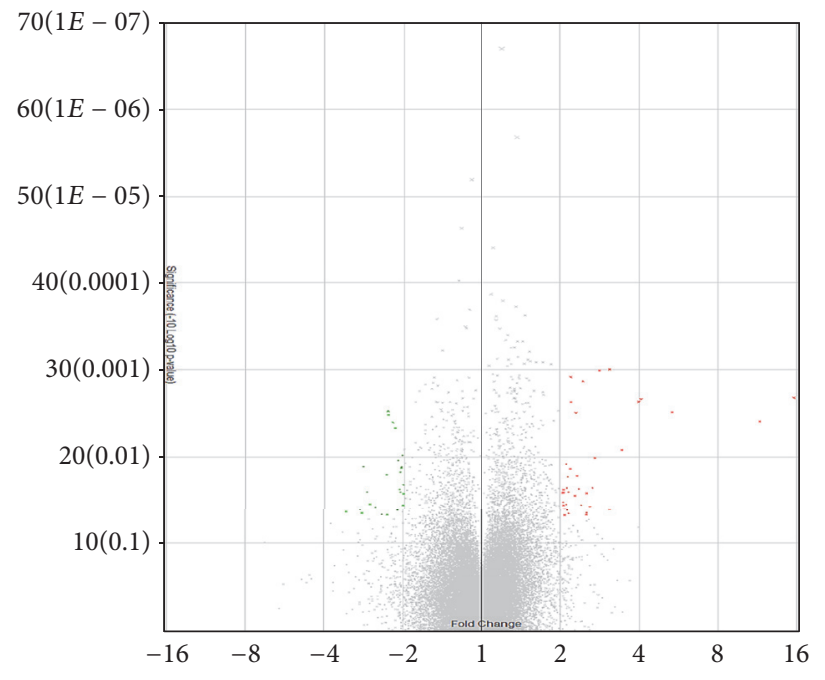

(a)

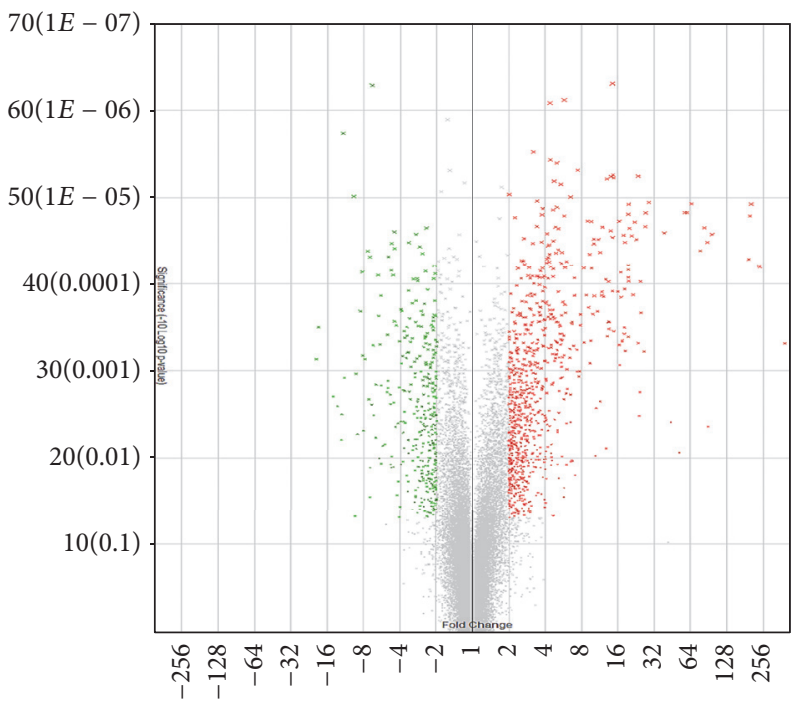

(b)

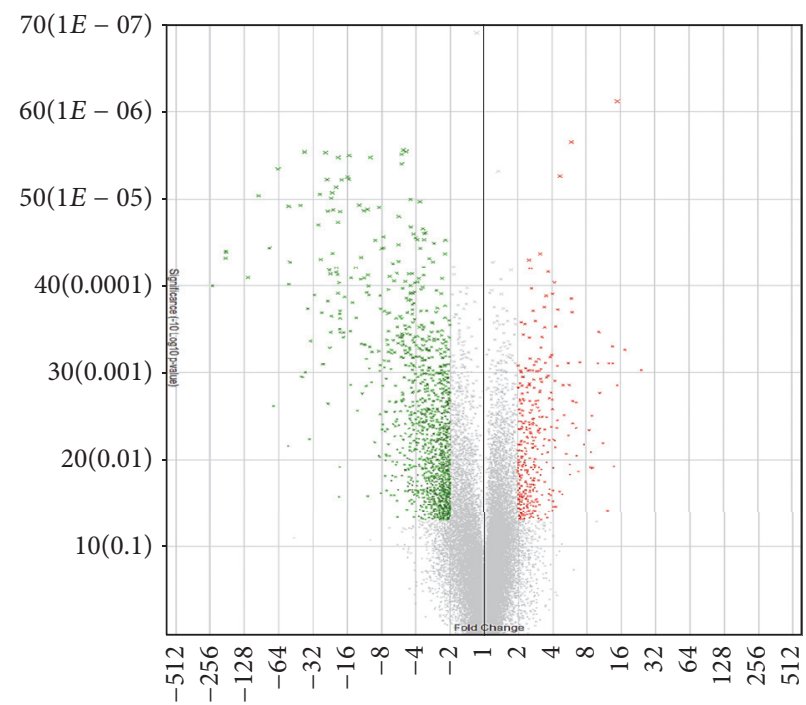

(c)

FIgURE 2: Volcano plots to identify changes in gene expression between (a) MSC-17 versus UT-MSC, (b) MSC- $\gamma$ versus UT-MSC, and (c) MSC-17 versus MSC- $\gamma$. Axes of these plots represent significance $(-10 \log 10 p$ value of the ANOVA $p$ values; $y$-axes) versus fold changes (linear fold change from condition pairing; $x$-axes). Red colour indicates upregulated genes and the green represents downregulated genes. The grey region indicates genes that were not differentially expressed and not statistically significant.

coherence with the observation of decreased MSC- $\gamma$ growth kinetics compared to UT-MSC [1].

Gene ontology analysis for cellular components (Tables S7, S8) of the differentially regulated genes in the MSC$\gamma$ versus UT-MSC groups uncovered that these genes were located in the extracellular space (or) region (annotation cluster 1, enrichment score 2.69, Table S7; enrichment score 12.76, Table S8). Many downregulated genes were located in collagen, the main structural protein in the extracellular region.

3.3. MSC-17 Enriched for Genes Associated with Chemotaxis. Differentially regulated genes were submitted to DAVID for functional annotation clustering to identify gene sets that were enriched in MSC-17. Genes that were mapped by DAVID were shown in Table 2. There were 23 genes from the gene entry list that were unmapped by DAVID (Table S9). These include noncoding genes, lncRNA, ribosomal RNA (rRNA), snoRNA, and miRNA.

Functional annotation clustering analysis was first performed using DAVID's default settings (Table S10) to identify overall gene sets that were highly enriched in MSC17 compared to UT-MSC. Annotation cluster 1 with the highest enrichment score (3.58) had enriched terms for genes residing in the extracellular region, roles in inflammatory response, response to wounding, defense responses, signaling, and disulfide bonds (Table S10). Gene ontology 


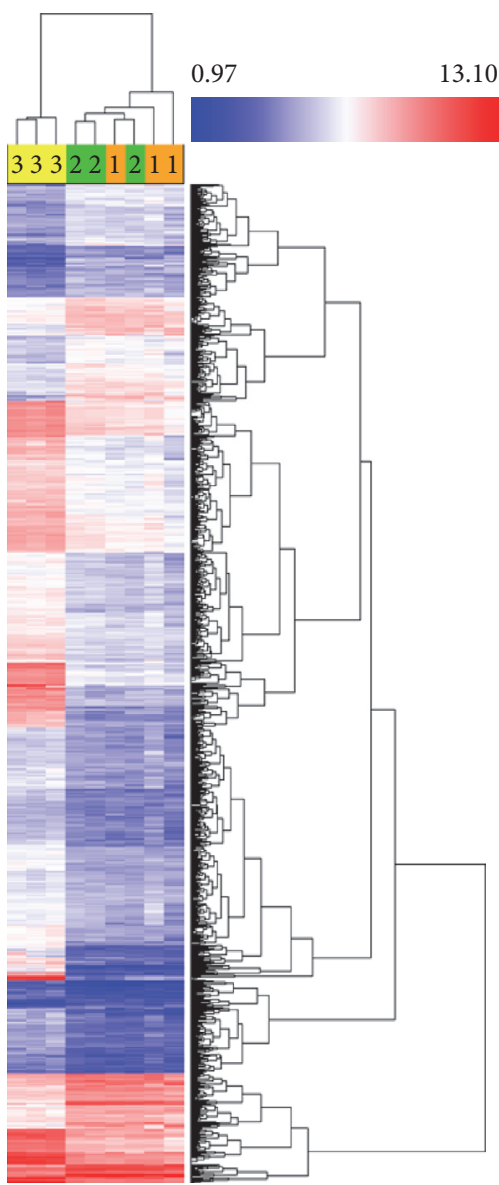

(a)

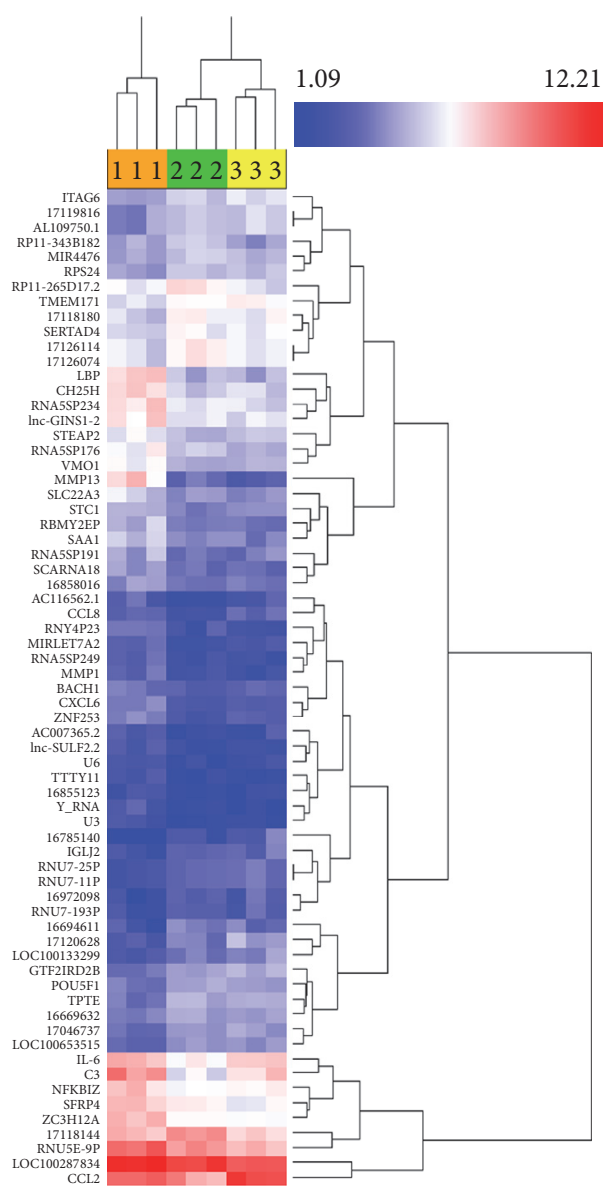

(b)

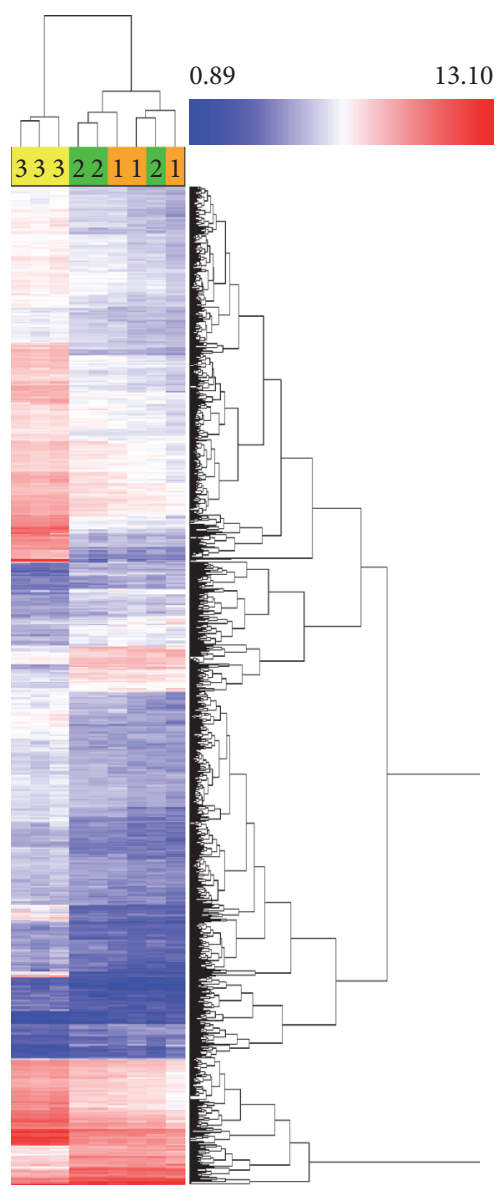

(c)

Figure 3: Gene expression profile of MSC-17 (1), UT-MSC (2), and MSC- $\gamma$ (3) from 3 MSC donors determined with Affymetrix Human Gene ST 2.0 microarrays. Supervised hierarchical clustering of genes differentially expressed between (a) MSC- $\gamma$ versus UT-MSC, (b) MSC17 versus UT-MSC, and (c) MSC-17 versus MSC- $\gamma$ determined by ANOVA $p$ value (condition pair) $p<0.05$ and fold change (linear) $<-2$ or $>2$. (a) 1278 and (b) 67 genes were differentially regulated between the treatment groups. The normalized expression value for each gene is visualised by a colour gradient: blue represents low gene expression; red represents high gene expression.

analysis of biological processes also revealed that MSC-17 compared to MSC- $\gamma$ were upregulated and enriched for genes involved in angiogenesis (e.g., angiogenin, CXCL12, tissue plasminogen activator, and collagens), wound healing, and chemotaxis responses (Table S14). Interestingly, some gene sets were enriched for gene ontology terms such as glycosylation and glycoproteins (Table S10), which may relate to posttranslational modification processes [26, 27]. There was also high enrichment of genes involved in chromatin remodelling processes (enrichment score 7.35, Table S14), suggesting the potential gene expression regulatory roles of MSC-17.

Human MSC-17 were shown to be superior at regulating $\mathrm{T}$ cell inflammatory responses by suppressing $\mathrm{T}$ cell proliferation, activation, and secretion of proinflammatory cytokines [1]. In annotation cluster 3 (enrichment score 2.48, Table S10), genes such as IL-6, C3, serum amyloid A1 (SAA1), and lipopolysaccharide binding protein (LBP) were enriched for regulation of immune responses. IL-6, SAA1, and LBP also have roles in regulation of cytokine production.
Gene ontology analysis by DAVID functional annotation clustering was also performed on the MSC-17 versus UTMSC and MSC-17 versus MSC- $\gamma$ gene lists to specifically determine enriched gene sets for biological processes (Tables S11, S14, and S15), molecular functions (Tables S12, S16), and cellular components (Tables S13, S17, and S18) in MSC17. There was no significant enrichment of gene sets for molecular functions in the downregulated gene list of MSC17 versus MSC- $\gamma$ comparison group.

MSC-17 were previously shown to mediate Treg induction via cell-cell contact dependent mechanisms [1]. To identify potential cell surface candidate molecules that mediate MSC17 induction of Tregs, the cellular compartments of genes enriched in the MSC-17 were also evaluated. Functional enrichment for biological processes identified a set of upregulated genes (IL-6, CCL8, SLC22A3, STC1, and CXCL6) that were enriched for the gene ontology term cell-cell signaling (fold enrichment: 4.9; $p<0.0148$; annotation cluster 2, Table S11). Chemokines CCL2, CCL8, and CXCL6 detected by DAVID's functional enrichment for molecular function 
(Table S12) showed evidence that these gene sets have a different range of binding potential including chemokine receptor, heparin, glycosaminoglycan, pattern, and polysaccharide binding activities. These MSC-17 enriched genes, mainly the chemokines and MMPs, were located in the extracellular space (or) region (Table S13).

Biological processes (GOTERM_BP_FAT; Tables S11, S14) and molecular functions (GOTERM_MF_FAT; Table S12) of MSC-17 enriched genes were mainly associated with cell migration and chemotaxis responses. MMPs were also highly enriched in the MSC-17 groups. Specifically, MMP13 (FC 15.6) and MMP1 (FC 2.4) were induced in the MSC-17 groups as detected by microarray gene expression analysis (Tables 2 and 3). DAVID's bioinformatics analysis revealed that these genes were enriched for gene ontology terms such as secreted, extracellular space, signal, disulfide bond, glycosylation, glycoproteins, and response to stimulus (Table S10). The MSC-17 versus UT-MSC gene list when analysed by DAVID functional annotation chart (default setting) showed that these MMPs where highly enriched for metal ion binding, peptidase, and collagen degradation functions (Table S10).

3.4. MSC-17 Express Chemokines and Matrix Metalloproteinases. To validate the microarray data of MSC-17, upregulated genes were evaluated for their gene expression by RTPCR (Figure 4). IL-17A induced the expression of MMP1, MMP13, CXCL6, C3, CH25H, and LBP in MSC as determined by microarray and validated by RT-PCR $(p<0.05)$. CCL2 and CCL8 were highly expressed in both MSC- $\gamma$ and MSC-17 compared to UT-MSC, consistent with the microarray data. CCL2 gene expression increased by 8.2- and 5.9fold in MSC- $\gamma$ and MSC-17, respectively, relative to UT-MSC. CCL8 expression on the other hand was comparable between MSC- $\gamma$ and MSC-17. Although the gene expression levels varied between the 3 MSC donors, these genes were consistently upregulated relative to UT-MSC in all the MSC donors.

\section{Discussion}

IFN- $\gamma$ preactivation of human MSC induced the expression of various immunoregulatory molecules including IDO, TLR3/4, IL-6, and PD-L1 that may enhance the inhibitory activity of MSC- $\gamma$ to mediate T cell suppression. IDO is a well characterised immunosuppressive molecule expressed by MSC upon induction with IFN- $\gamma$ [1, 28-30]. Administration of IDO deficient MSC (IDO ${ }^{-/-}$MSC) or inhibition of IDO activity resulted in accelerated kidney allograft rejection, decreased intragraft, or circulating Tregs and showed absence of donor-specific tolerance [29]. IDO ${ }^{-/-} \mathrm{MSC}$ were also incapable of inhibiting donor DC maturation and function, thus enabling $\mathrm{DC}$ to stimulate strong recipient $\mathrm{T}$ cell proliferative responses [29]. Consistent with previous literature [28, 29, 31], gene expression analysis revealed that IDO was the most highly induced gene in MSC- $\gamma$ and may be the key candidate molecule by which MSC- $\gamma$ mediate enhanced T cell immunosuppression [1].

MSC constitutively express a range of TLRs, including TLR3 and TLR4 [32, 33]. Activation of TLR3 and (or)
TLR4 amplifies MSC trophic factors, antimicrobial activity, and immunosuppressive potential, thereby enhancing MSC therapeutic potency [33-36]. Both TLR3 and TLR4 were upregulated in MSC- $\gamma$ compared to UT-MSC. Activation of TLR3 and TLR4 signaling with poly I:C or LPS, respectively, induced IDO expression in MSC [33]. TLR-driven induction of IDO in MSC resulted in the degradation of tryptophan and production of immunosuppressive kynurenines [33]. TLR3 activation has also been linked to expression of IL-6 in MSC [34]. IL-6 mediates the inhibitory effects of MSC on DC differentiation, maturation, and function [37, 38]. Consistent with our previous report, the upregulation of IL- 6 transcripts in MSC- $\gamma$ and high protein concentrations of IL- 6 in MSC- $\gamma$ T cell coculture supernatants suggest that MSC- $\gamma$ secreted IL6 may be involved in suppression of proinflammatory T cell responses [1]. Nevertheless, TLR activation in MSC is known to abrogate their immunosuppressive properties $[39,40]$. The effects of TLR signaling in MSC are still not fully understood and remain to be further investigated.

TLR3/4 preactivated MSC have enhanced leukocyte binding activity mediated by the induction of the adhesion molecule ICAM-1, consistent with upregulation of ICAM-1 in MSC- $\gamma$ [35]. ICAM-1 together with TLR3 and TLR4 were among the genes enriched for the gene ontology term positive regulation of immune system process, suggesting a potential biological role of TLR3/4 in MSC- $\gamma$ induction of ICAM-1 [35]. Additionally, the upregulation of chemokines such as CXCL9, CXCL10, CXCL11, CXCL16, CCL2, CCL8, and CCL13 detected by microarray may facilitate $\mathrm{T}$ cell recruitment to MSC- $\gamma$. Mouse MSC preactivated with IFN- $\gamma$ and TNF$\alpha$ induced CXCL9 and CXCL10 [41]. The production of these chemokines was abrogated by IFN $-\gamma$ neutralization [41]. Moreover, the blockade of CXCR3, a T cell receptor for chemokines CXCL9 and CXCL10, eliminated T cell chemotaxis towards MSC and subsequent MSC inhibition of $\mathrm{T}$ cell proliferation [41]. These studies concluded that cytokines induce MSC-expression of chemokines to drive $\mathrm{T}$ cell recruitment into close proximity with MSC, enabling MSC to suppress T cells through the secretion of immunosuppressive molecules [41, 42]. Chemokines also increased the in vivo migratory properties of human MSC- $\gamma$ to sites of inflammation in colitis mouse models [43]. Studies to validate the functional role of human MSC- $\gamma$ derived chemokines and ICAM-1 in the recruitment and subsequent modulation of $\mathrm{T}$ cell responses as well as in MSC $-\gamma$ homing to sites of inflammation in vivo are required. Hence, IFN- $\gamma$ directly induces an array of immunosuppressive molecules in MSC and may further amplify the secretion of other MSC-inhibitory molecules such as IDO, IL-6, and ICAM1 via TLR3/4 activation. MSC- $\gamma$ with higher proximity to leukocytes may serve as an additional mechanism by which MSC- $\gamma$ increase their modulatory activity on T cells.

Despite being highly immunosuppressive with enhanced homing and reparative capacities, allogeneic MSC- $\gamma$ are ineffective in vivo due to their increased immunogenicity [44-46]. A large number of genes upregulated in MSC- $\gamma$ were involved in the antigen processing and presentation pathways of MHC classes I and II. Antigen processing and presentation occurs via the cytosolic [47-51] or endocytic pathways 

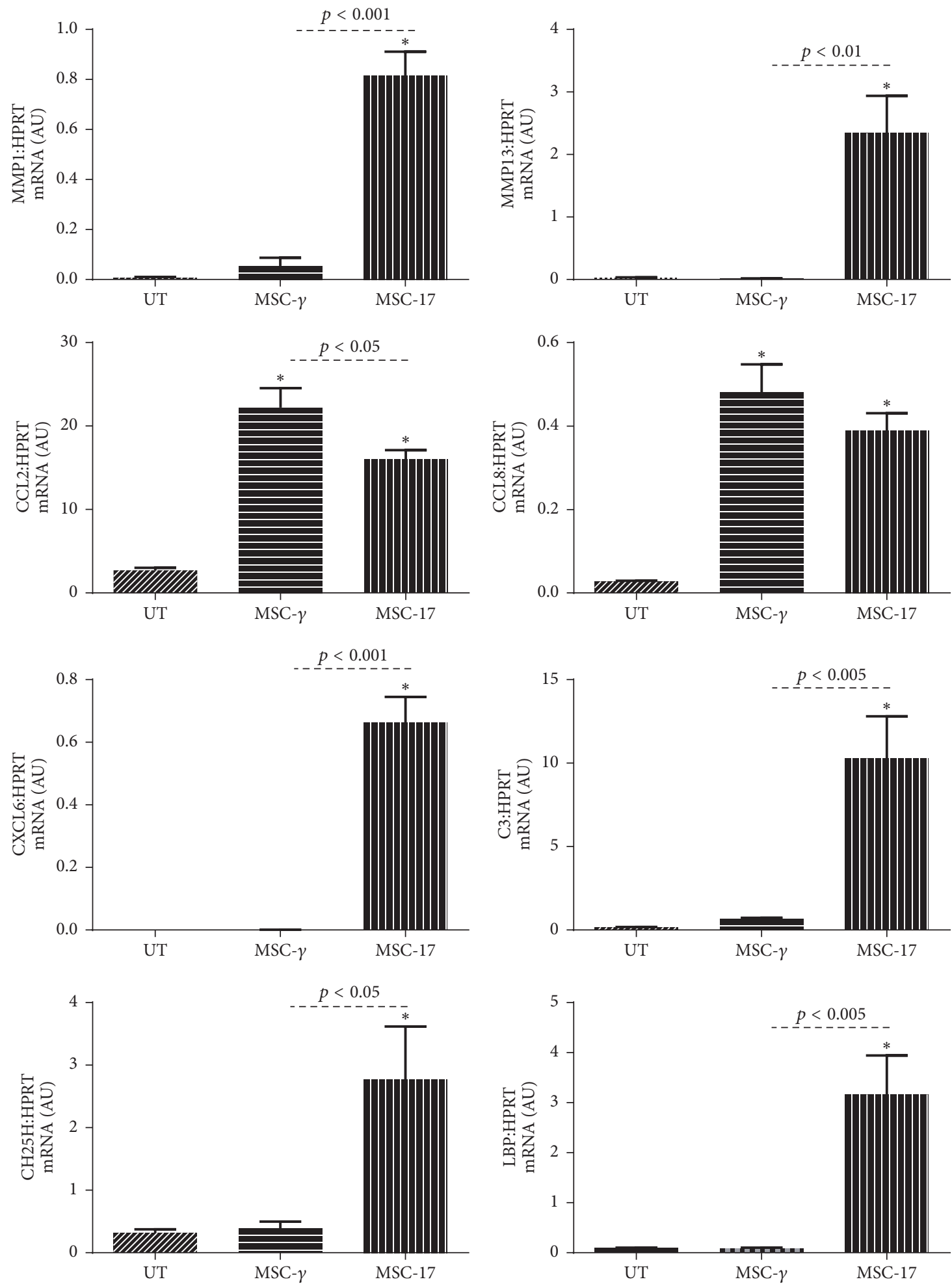

FIGURE 4: Microarray gene expression validation by RT-PCR. Gene expression of MMP1, MMP13, CCL2, CCL8, CXCL6, C3, LBP, and CH25 in MSC detected by microarray was validated by RT-PCR following 5 days of IL-17A or IFN- $\gamma$ treatment of human MSC. ${ }^{*} p<0.05$ versus UT-MSC was determined by one-way ANOVA with post-Sidak multiple comparison test. Data are representative of 3 human MSC donors. Error bars depict mean \pm SD. 
[51, 52]. In the cytosolic pathway, degraded intracellular proteins are transported to the rough endoplasmic reticulum (RER) via TAP, a heterodimer consisting of TAP1 and TAP2. These peptides are further trimmed by aminopeptidases ERAP to enable optimal peptide loading onto MHC class I molecules. MHC class I components comprise the class I MHC $\alpha$-chain and the B2M chain. This MHC class I molecule associates with the chaperone molecules tapasin, calreticulin, and ERp57. Tapasin (TAPBPL) recruits the MHC I molecules into proximity to TAP, allowing efficient peptide loading onto MHC class I molecules, subsequently stabilizing the peptideclass I molecule complex. The class I MHC-peptide complex is then transported to the plasma membrane for antigenpeptide presentation to $\mathrm{CD}^{+} \mathrm{T}$ cells [47-51]. Induction of ERAP, TAP2, TAPBPL, and B2M, genes involved in this cytosolic pathway was evident in MSC- $\gamma$ and correlated with the observed upregulation of MHC class I in these cells [1]. We have also shown that MHC class II is induced in MSC$\gamma$ [1]. In the endocytic pathway, assembly of MHC class II occurs in RER where the $\alpha$ - and $\beta$-chain associate and this newly synthesised class II MHC complex binds to the invariant chain (Ii, CD74). As MHC class II-Ii complex is translocated into the endosomal compartment, the Ii chain is degraded, leaving the CLIP fragment (class II associated Ii peptide) bound to the MHC II peptide binding cleft. HLADM catalyses the exchange of CLIP with the antigenic peptide. The MHC class II peptide complex is then transported to the plasma membrane for antigen presentation to $\mathrm{CD} 4^{+}$ $\mathrm{T}$ cells $[51,52]$. We detected high expression of CD74 and HLA-DM in MSC- $\gamma$, supporting the induction of MHC class II on these cells. Alloimmune responses against UT-MSC are mediated by the recognition of allogeneic MHC molecules by recipient $\mathrm{CD}^{+}$and $\mathrm{CD}^{+}$memory T cells [53]. MHC class II expression on allogeneic MSC is also known to induce alloimmune responses in cocultures with MHC-mismatched responder cells [54]. Therefore, the amplification of this antigen processing and presentation machinery suggests that MSC $-\gamma$ are highly immunogenic and can potently prime proinflammatory $\mathrm{T}$ cell responses in allogeneic hosts.

Moreover, MSC- $\gamma$ were enriched for gene sets involved in augmentation of the humoral immunity and complement pathways activation. Our data may explain previously published data, where MSC- $\gamma$ infused mice had higher levels of circulating anti-donor IgM and IgG alloantibodies, which resulted in the rapid induction of antibody-mediated rejection [44]. Although MSC- $\gamma$ lack the expression of costimulatory signals (CD80, CD83, and CD86) to function as APC to mediate direct $\mathrm{T}$ cell allorecognition and activation $[1,55-58]$, we speculate that MSC- $\gamma$ induce allogeneic T cell responses through the indirect or semidirect pathways of allorecognition [21, 59]. Allogeneic MHC-peptide transfer from MSC $-\gamma$ could be more rapid compared to UT-MSC due to high expression of MHC molecules. This enables allogeneic MHC-peptide to be recognised by recipient $\mathrm{T}$ cells through the semidirect pathway. Understanding mechanisms of MSC- $\gamma$ immunogenicity may enable the targeting of MSC through different pathways of activation to increase their immunomodulatory function whilst retaining MSC in a nonimmunogenic and inert state.
Human MSC-17 showed superior suppression of T cell responses and were able to induce Tregs with minimal immunogenicity [1]. MSC constitutively express a range of MMPs including MMP2, membrane type 1 MMP (MTIMMP), tissue inhibitor of MMP1 (TIMP1), and TIMP2 [60-62]. These MMPs are essential for MSC invasion and migration across the extracellular matrix (ECM) as demonstrated in in vitro transendothelial migration assays [62]. Absence of MMPs impairs MSC transmigration capacity across Matrigels [60-62]. In response to IL-1 $\beta$ and TNF$\alpha$, MSC have also been shown to amplify the expression of MMP2, MTI-MMP, and (or) MMP9 in MSC, thereby promoting MSC invasiveness across the basement membrane [60]. Here, we demonstrated that IL-17A induced the gene expression of MMP13 and MMP1 in MSC. These MMPs were highly enriched for collagen degradation and metabolic processes, suggesting that these factors may be essential for MSC-17 to invade the ECM.

MSC-derived MMPs also have proteolytic activity on chemokines [63, 64]. MMP processing of CC chemokines convert the biochemical properties of the chemokine target molecules from an agonist to an antagonist form with antiinflammatory properties in vivo [65]. MSC-derived MMP1 cleaves CCL2, leading to the generation of CCL2 with suppressive properties on $\mathrm{B}$ cell production of immunoglobulins and in $\mathrm{CD}^{+} \mathrm{T}$ cell activation $[63,64]$. We showed that MMP1, CCL2, and MMP13 were upregulated in human MSC-17. Evaluating the functional role of MMP-processed chemokine derivatives in MSC-17 immunomodulation on T cells in this study remains to be elucidated.

MMP-2 and MMP-9 secreted by MSC are known to cleave and reduce $\mathrm{CD} 25$ expression on $\mathrm{T}$ cells, thus impairing $\mathrm{T}$ cell activation and proliferation [66]. Administration of MMP inhibitors in an islet allotransplant model abrogated the suppressive effect of MSC on alloreactive T cells, resulting in allograft rejection. This study concluded that MMPs are crucial for MSC immunosuppression [66]. We have previously shown that MSC-17 further downregulated CD25 expression on $\mathrm{CD}^{+}$effector $\mathrm{T}$ cells compared to UT-MSC, a process partially mediated by cell contact dependent mechanisms [1]. The involvement of MSC-17-derived MMP13 and MMP1 in downregulating $\mathrm{CD} 25$ on $\mathrm{T}$ cells has not been previously established. Blocking MMP13 activity using specific inhibitors may provide insights on its role in inhibiting $\mathrm{T}$ cell activation.

Apart from the upregulation of chemotactic transcripts, MSC-17 were also enriched for genes involved in wound healing and angiogenesis. Tissue plasminogen activator (PLAT) was upregulated in human bone marrow derived MSC-17 when compared to MSC- $\gamma$. PLAT was enriched for biological processes involving cell motility, angiogenesis, and responses to wounding. A previous study reported that IL-17A can increase MSC migration in an in vitro wound healing assay [7]. In a latter study, IL-17A was shown to enhance peripheral blood-derived MSC migration in a wound healing assay by inducing the expression of the urokinase type plasminogen activator through the activation of ERK1,2-MAPK signaling pathway [67]. Increased expression of the urokinase type plasminogen activator has been reported to facilitate MSC 
transendothelial migration, potentially contributing to MSC motility to sites of inflammation for tissue regeneration or immunosuppression [67]. In other studies, tissue plasminogen activators have also shown to support angiogenesis by promoting vascular endothelial cell migration to ischemic regions $[68,69]$. These data suggest that MSC-17 in addition to their potent immunosuppressive properties may benefit disease conditions of ischemia injury that require tissue repair and angiogenesis.

In this study, donor to donor variation may have limited the robustness of our microarray data to detect subtle changes in MSC-17 gene expression profile. However, real-time PCR data validated changes detected in the highly regulated genes in MSC-17. More MSC-17 biological replicates may provide further insights into other genes that are differently regulated.

\section{Conclusions}

Enhanced expression of MHC in allogeneic MSC- $\gamma$ increases their immunogenicity and this may negatively impact MSC$\gamma$ potency in vivo. Nevertheless, we have highlighted novel candidate immunosuppressive molecules and pathways in which MSC- $\gamma$ can be targeted in future studies to increase the immunomodulatory capacity of MSC. We have also identified a few novel candidate molecules that may contribute to the potent MSC-17 regulation of immune responses. These candidate molecules can be explored for their regulatory roles in MSC-17 suppression of T cell responses and in the generation of Tregs in future studies.

\section{Disclosure}

An earlier version of this work was presented as an abstract at the Transplantation Science Symposium, 2015, and at the American Transplant Congress, 2016.

\section{Competing Interests}

The authors declare no competing financial interests.

\section{Acknowledgments}

The authors thank the Adelaide Microarray Centre, University of Adelaide, for running the microarray samples. They also thank Dr. Philip Gregory, SA Pathology, South Australia, for proof-reading and providing feedback on this manuscript. They also thank Svjetlana Kireta and Julie Johnston for proof-reading this manuscript. This work was supported by grants from The Hospital Research Foundation, The Queen Elizabeth Hospital, Adelaide, South Australia. Kisha Nandini Sivanathan, Ph.D., was financed by the Adelaide Graduate Research Scholarship (University of Adelaide, South Australia) and The Hospital Research Foundation Scholarship (The Queen Elizabeth Hospital, Adelaide, South Australia). Kisha Nandini Sivanathan also received a young investigator grant from the 2015 Transplantation Science Symposium for this work.

\section{References}

[1] K. N. Sivanathan, D. M. Rojas-Canales, C. M. Hope et al., "Interleukin-17A-induced human mesenchymal stem cells are superior modulators of immunological function," Stem Cells, vol. 33, no. 9, pp. 2850-2863, 2015.

[2] S. Ivanov and A. Lindén, "Interleukin-17 as a drug target in human disease," Trends in Pharmacological Sciences, vol. 30, no. 2, pp. 95-103, 2009.

[3] Y. Iwakura, H. Ishigame, S. Saijo, and S. Nakae, "Functional specialization of interleukin-17 family members," Immunity, vol. 34, no. 2, pp. 149-162, 2011.

[4] X. Song and Y. Qian, "The activation and regulation of IL-17 receptor mediated signaling," Cytokine, vol. 62, no. 2, pp. 175182, 2013.

[5] H. C. Seon, H. Park, and C. Dong, "Actl adaptor protein is an immediate and essential signaling component of interleukin-17 receptor," Journal of Biological Chemistry, vol. 281, no. 47, pp. 35603-35607, 2006.

[6] W. Huang, V. La Russa, A. Alzoubi, and P. Schwarzenberger, "Interleukin-17A: a T-cell-derived growth factor for murine and human mesenchymal stem cells," Stem Cells, vol. 24, no. 6, pp. 1512-1518, 2006.

[7] H. Huang, H. J. Kim, E.-J. Chang et al., "IL-17 stimulates the proliferation and differentiation of human mesenchymal stem cells: implications for bone remodeling," Cell Death and Differentiation, vol. 16, no. 10, pp. 1332-1343, 2009.

[8] S. Mojsilović, A. Krstić, V. Ilić et al., "IL-17 and FGF signaling involved in mouse mesenchymal stem cell proliferation," Cell and Tissue Research, vol. 346, no. 3, pp. 305-316, 2011.

[9] K. Schroder, P. J. Hertzog, T. Ravasi, and D. A. Hume, "Interferon- $\gamma$ : an overview of signals, mechanisms and functions," Journal of Leukocyte Biology, vol. 75, no. 2, pp. 163-189, 2004.

[10] L. C. Platanias, "Mechanisms of type-I- and type-II-interferonmediated signalling," Nature Reviews Immunology, vol. 5, no. 5, pp. 375-386, 2005.

[11] H. A. Young and K. J. Hardy, "Role of interferon-gamma in immune cell regulation," Journal of Leukocyte Biology, vol. 58, no. 4, pp. 373-381, 1995.

[12] S. D. Der, A. Zhou, B. R. G. Williams, and R. H. Silverman, "Identification of genes differentially regulated by interferon $\alpha$, $\beta$, or $\gamma$ using oligonucleotide arrays," Proceedings of the National Academy of Sciences of the United States of America, vol. 95, no. 26, pp. 15623-15628, 1998.

[13] J. L. Pace, S. W. Russell, B. A. Torres, H. M. Johnson, and P. W. Gray, "Recombinant mouse $\gamma$ interferon induces the priming step in macrophage activation for tumor cell killing," Journal of Immunology, vol. 130, no. 5, pp. 2011-2013, 1983.

[14] C. F. Nathan, H. W. Murray, M. E. Wiebe, and B. Y. Rubin, "Identification of interferon- $\gamma$ as the lymphokine that activates human macrophage oxidative metabolism and antimicrobial activity," Journal of Experimental Medicine, vol. 158, no. 3, pp. 670-689, 1983.

[15] T. Y. Basham and T. C. Merigan, "Recombinant interferon$\gamma$ increases HLA-DR synthesis and expression," Journal of Immunology, vol. 130, no. 4, pp. 1492-1494, 1983.

[16] U. Boehm, T. Klamp, M. Groot, and J. C. Howard, "Cellular responses to interferon- $\gamma$," Annual Review of Immunology, vol. 15, pp. 749-795, 1997. 
[17] T. D. Geppert and P. E. Lipsky, "Antigen presentation by interferon- $\gamma$-treated endothelial cells and fibroblasts: differential ability to function as antigen-presenting cells despite comparable Ia expression," Journal of Immunology, vol. 135, no. 6, pp. 3750-3762, 1985.

[18] F. D. Finkelman, I. M. Katona, T. R. Mosmann, and R. L. Coffman, "IFN- $\gamma$ regulates the isotypes of Ig secreted during in vivo humoral immune responses," The Journal of Immunology, vol. 140, no. 4, pp. 1022-1027, 1988.

[19] A. O'Garra, "Cytokines induce the development of functionally heterogeneous T helper cell subsets," Immunity, vol. 8, no. 3, pp. 275-283, 1998.

[20] A. Le Moine, M. Goldman, and D. Abramowicz, "Multiple pathways to allograft rejection," Transplantation, vol. 73, no. 9, pp. 1373-1381, 2002.

[21] K. N. Sivanathan, S. Gronthos, D. Rojas-Canales, B. Thierry, and P. T. Coates, "Interferon-gamma modification of mesenchymal stem cells: implications of autologous and allogeneic mesenchymal stem cell therapy in allotransplantation," Stem Cell Reviews and Reports, vol. 10, no. 3, pp. 351-375, 2014.

[22] S. Gronthos, S. E. Graves, S. Ohta, and P. J. Simmons, "The STRO-1+ fraction of adult human bone marrow contains the osteogenic precursors," Blood, vol. 84, no. 12, pp. 4164-4173, 1994.

[23] P. J. Simmons, S. Gronthos, A. Zannettino, S. Ohta, and S. Graves, "Isolation, characterization and functional activity of human marrow stromal progenitors in hemopoiesis," Progress in Clinical and Biological Research, vol. 389, pp. 271-280, 1994.

[24] D. W. Huang, B. T. Sherman, and R. A. Lempicki, "Systematic and integrative analysis of large gene lists using DAVID bioinformatics resources," Nature Protocols, vol. 4, no. 1, pp. 44-57, 2009.

[25] P. Y. Muller, H. Janovjak, A. R. Miserez, and Z. Dobbie, "Processing of gene expression data generated by quantitative real-time RT-PCR," BioTechniques, vol. 32, no. 6, pp. 1372-1379, 2002.

[26] F. Wold, "In vivo chemical modification of proteins (posttranslational modification)," Annual Review of Biochemistry, vol. 50, pp. 783-814, 1981.

[27] C. T. Walsh, S. Garneau-Tsodikova, and G. J. Gatto Jr., "Protein posttranslational modifications: the chemistry of proteome diversifications," Angewandte Chemie-International Edition, vol. 44, no. 45, pp. 7342-7372, 2005.

[28] R. Meisel, A. Zibert, M. Laryea, U. Göbel, W. Däubener, and D. Dilloo, "Human bone marrow stromal cells inhibit allogeneic T-cell responses by indoleamine 2,3-dioxygenasemediated tryptophan degradation," Blood, vol. 103, no. 12, pp. 4619-4621, 2004.

[29] W. Ge, J. Jiang, J. Arp, W. Liu, B. Garcia, and H. Wang, "Regulatory T-cell generation and kidney allograft tolerance induced by mesenchymal stem cells associated with indoleamine 2,3dioxygenase expression," Transplantation, vol. 90, no. 12, pp. 1312-1320, 2010.

[30] N. Wada, P. M. Bartold, and S. Gronthos, "Human foreskin fibroblasts exert immunomodulatory properties by a different mechanism to bone marrow stromal/stem cells," Stem Cells and Development, vol. 20, no. 4, pp. 647-659, 2011.

[31] M. François, R. Romieu-Mourez, M. Li, and J. Galipeau, "Human MSC suppression correlates with cytokine induction of indoleamine 2,3-dioxygenase and bystander M2 macrophage differentiation," Molecular Therapy, vol. 20, no. 1, pp. 187-195, 2012.
[32] X.-X. He, H. Bai, G.-R. Yang, Y.-J. Xue, and Y.-N. Su, "Expression of Toll-like receptors in human bone marrow mesenchymal stem cells," Journal of Experimental Hematology, vol. 17, no. 3, pp. 695-699, 2009.

[33] C. A. Opitz, U. M. Litzenburger, C. Lutz et al., “Toll-like receptor engagement enhances the immunosuppressive properties of human bone marrow-derived mesenchymal stem cells by inducing indoleamine-2,3-dioxygenase- 1 via Interferon- $\beta$ and protein kinase R," Stem Cells, vol. 27, no. 4, pp. 909-919, 2009.

[34] M. Mastri, Z. Shah, T. McLaughlin et al., "Activation of tolllike receptor 3 amplifies mesenchymal stem cell trophic factors and enhances therapeutic potency," American Journal of Physiology-Cell Physiology, vol. 303, no. 10, pp. C1021-C1033, 2012.

[35] D. J. Kota, B. Dicarlo, R. A. Hetz, P. Smith, C. S. Cox Jr., and S. D. Olson, "Differential MSC activation leads to distinct mononuclear leukocyte binding mechanisms," Scientific Reports, vol. 4, article no. 4565, 2014.

[36] D. K. Sung, Y. S. Chang, S. I. Sung, H. S. Yoo, S. Y. Ahn, and W. S. Park, "Antibacterial effect of mesenchymal stem cells against Escherichia coli is mediated by secretion of beta- defensin- 2 via toll- like receptor 4 signalling," Cellular Microbiology, vol. 18, no. 3, pp. 424-436, 2016.

[37] F. Djouad, L.-M. Charbonnier, C. Bouffi et al., "Mesenchymal stem cells inhibit the differentiation of dendritic cells through an interleukin-6-dependent mechanism," Stem Cells, vol. 25, no. 8, pp. 2025-2032, 2007.

[38] H.-Y. Lai, M.-J. Yang, K.-C. Wen, K.-C. Chao, C.-C. Shih, and O. K. Lee, "Mesenchymal stem cells negatively regulate dendritic lineage commitment of umbilical-cord-bloodderived hematopoietic stem cells: an unappreciated mechanism as immunomodulators," Tissue Engineering-Part A, vol. 16, no. 9, pp. 2987-2997, 2010.

[39] G. Raicevic, M. Najar, B. Stamatopoulos et al., "The source of human mesenchymal stromal cells influences their TLR profile as well as their functional properties," Cellular Immunology, vol. 270, no. 2, pp. 207-216, 2011.

[40] J. Lei, Z. Wang, D. Hui et al., "Ligation of TLR2 and TLR4 on murine bonTritschler I.e marrow-derived mesenchymal stem cells triggers differential effects on their immunosuppressive activity," Cellular Immunology, vol. 271, no. 1, pp. 147-156, 2011.

[41] G. Ren, L. Zhang, X. Zhao et al., "Mesenchymal stem cellmediated immunosuppression occurs via concerted action of chemokines and nitric oxide," Cell Stem Cell, vol. 2, no. 2, pp. 141-150, 2008.

[42] Y. Shi, G. Hu, J. Su et al., "Mesenchymal stem cells: a new strategy for immunosuppression and tissue repair," Cell Research, vol. 20, no. 5, pp. 510-518, 2010.

[43] M. Duijvestein, M. E. Wildenberg, M. M. Welling et al., "Pretreatment with interferon- $\gamma$ enhances the therapeutic activity of mesenchymal stromal cells in animal models of colitis," Stem Cells, vol. 29, no. 10, pp. 1549-1558, 2011.

[44] A. T. Badillo, K. J. Beggs, E. H. Javazon, J. C. Tebbets, and A. W. Flake, "Murine bone marrow stromal progenitor cells elicit an in vivo cellular and humoral alloimmune response," Biology of Blood and Marrow Transplantation, vol. 13, no. 4, pp. 412-422, 2007.

[45] M. Rafei, E. Birman, K. Forner, and J. Galipeau, "Allogeneic mesenchymal stem cells for treatment of experimental autoimmune encephalomyelitis," Molecular Therapy, vol. 17, no. 10, pp. 1799-1803, 2009. 
[46] S. Schu, M. Nosov, L. O’Flynn et al., "Immunogenicity of allogeneic mesenchymal stem cells," Journal of Cellular and Molecular Medicine, vol. 16, no. 9, pp. 2094-2103, 2012.

[47] P. J. Lehner and P. Cresswell, "Processing and delivery of peptides presented by MHC class I molecules," Current Opinion in Immunology, vol. 8, no. 1, pp. 59-67, 1996.

[48] I. A. York, A. L. Goldberg, X. Y. Mo, and K. L. Rock, "Proteolysis and class I major histocompatibility complex antigen presentation," Immunological Reviews, vol. 172, pp. 49-66, 1999.

[49] J. Koch, R. Guntrum, S. Heintke, C. Kyritsis, and R. Tampé, "Functional dissection of the transmembrane domains of the transporter associated with antigen processing (TAP)," Journal of Biological Chemistry, vol. 279, no. 11, pp. 10142-10147, 2004.

[50] K. L. Rock, I. A. York, and A. L. Goldberg, "Post-proteasomal antigen processing for major histocompatibility complex class I presentation," Nature Immunology, vol. 5, no. 7, pp. 670-677, 2004.

[51] J. Neefjes, M. L. M. Jongsma, P. Paul, and O. Bakke, "Towards a systems understanding of MHC class I and MHC class II antigen presentation," Nature Reviews Immunology, vol. 11, no. 12, pp. 823-836, 2011.

[52] S. J. Turley, K. Inaba, W. S. Garrett et al., "Transport of peptideMHC class II complexes in developing dendritic cells," Science, vol. 288, no. 5465, pp. 522-527, 2000.

[53] L. Zangi, R. Margalit, S. Reich-Zeliger et al., "Direct imaging of immune rejection and memory induction by allogeneic mesenchymal stromal cells," Stem Cells, vol. 27, no. 11, pp. 28652874, 2009.

[54] J. A. Potian, H. Aviv, N. M. Ponzio, J. S. Harrison, and P. Rameshwar, "Veto-like activity of mesenchymal stem cells: functional discrimination between cellular responses to alloantigens and recall antigens," Journal of Immunology, vol. 171, no. 7, pp. 3426-3434, 2003.

[55] M. Krampera, S. Glennie, J. Dyson et al., "Bone marrow mesenchymal stem cells inhibit the response of naive and memory antigen-specific T cells to their cognate peptide," Blood, vol. 101, no. 9, pp. 3722-3729, 2003.

[56] P. Batten, P. Sarathchandra, J. W. Antoniw et al., "Human mesenchymal stem cells induce $\mathrm{T}$ cell anergy and downregulate T cell allo-responses via the $\mathrm{TH} 2$ pathway: relevance to tissue engineering human heart valves," Tissue Engineering, vol. 12, no. 8, pp. 2263-2273, 2006.

[57] S. J. Prasanna, D. Gopalakrishnan, S. R. Shankar, and A. B. Vasandan, "Pro-inflammatory cytokines, IFN $\gamma$ and TNF $\alpha$, influence immune properties of human bone marrow and Wharton jelly mesenchymal stem cells differentially," PLoS ONE, vol. 5, no. 2, Article ID e9016, 2010.

[58] S. Aggarwal and M. F. Pittenger, "Human mesenchymal stem cells modulate allogeneic immune cell responses," Blood, vol. 105, no. 4, pp. 1815-1822, 2005.

[59] M. D. Griffin, A. E. Ryan, S. Alagesan, P. Lohan, O. Treacy, and T. Ritter, "Anti-donor immune responses elicited by allogeneic mesenchymal stem cells: what have we learned so far," Immunology and Cell Biology, vol. 91, no. 1, pp. 40-51, 2013.

[60] C. Ries, V. Egea, M. Karow, H. Kolb, M. Jochum, and P. Neth, "MMP-2, MT1-MMP, and TIMP-2 are essential for the invasive capacity of human mesenchymal stem cells: differential regulation by inflammatory cytokines," Blood, vol. 109, no. 9, pp. 4055-4063, 2007.

[61] A. De Becker, P. Van Hummelen, M. Bakkus et al., "Migration of culture-expanded human mesenchymal stem cells through bone marrow endothelium is regulated by matrix metalloproteinase- 2 and tissue inhibitor of metalloproteinase3," Haematologica, vol. 92, no. 4, pp. 440-449, 2007.

[62] T. Tondreau, N. Meuleman, B. Stamatopoulos et al., "In vitro study of matrix metalloproteinase/tissue inhibitor of metalloproteinase production by mesenchymal stromal cells in response to inflammatory cytokines: the role of their migration in injured tissues," Cytotherapy, vol. 11, no. 5, pp. 559-569, 2009.

[63] M. Rafei, J. Hsieh, S. Fortier et al., "Mesenchymal stromal cell derived CCL2 suppresses plasma cell immunoglobulin production via STAT3 inactivation and PAX5 induction," Blood, vol. 112, no. 13, pp. 4991-4998, 2008.

[64] M. Rafei, P. M. Campeau, A. Aguilar-Mahecha et al., "Mesenchymal stromal cells ameliorate experimental autoimmune encephalomyelitis by inhibiting CD4 Th17 T cells in a CC chemokine ligand 2-dependent manner," Journal of Immunology, vol. 182, no. 10, pp. 5994-6002, 2009.

[65] G. Angus McQuibban, J.-H. Gong, J. P. Wong, J. L. Wallace, I. Clark-Lewis, and C. M. Overall, "Matrix metalloproteinase processing of monocyte chemoattractant proteins generates CC chemokine receptor antagonists with anti-inflammatory properties in vivo," Blood, vol. 100, no. 4, pp. 1160-1167, 2002.

[66] Y. Ding, D. Xu, G. Feng, A. Bushell, R. J. Muschel, and K. J. Wood, "Mesenchymal stem cells prevent the rejection of fully allogenic islet grafts by the immunosuppressive activity of matrix metalloproteinase-2 and -9," Diabetes, vol. 58, no. 8, pp. 1797-1806, 2009.

[67] J. Krstić, H. Obradović, A. Jauković et al., "Urokinase type plasminogen activator mediates Interleukin-17-induced peripheral blood mesenchymal stem cell motility and transendothelial migration," Biochimica et Biophysica Acta, vol. 1853, no. 2, pp. 431-444, 2015.

[68] H.-K. Yip, C.-K. Sun, T.-H. Tsai et al., “Tissue plasminogen activator enhances mobilization of endothelial progenitor cells and angiogenesis in murine limb ischemia," International Journal of Cardiology, vol. 168, no. 1, pp. 226-236, 2013.

[69] V. Stepanova, P.-S. Jayaraman, S. V. Zaitsev et al., "Urokinasetype plasminogen activator (uPA) promotes angiogenesis by attenuating proline-rich homeodomain protein $(\mathrm{PRH})$ transcription factor activity and de-repressing vascular endothelial growth factor (VEGF) receptor expression," Journal of Biological Chemistry, vol. 291, no. 29, pp. 15029-15045, 2016. 

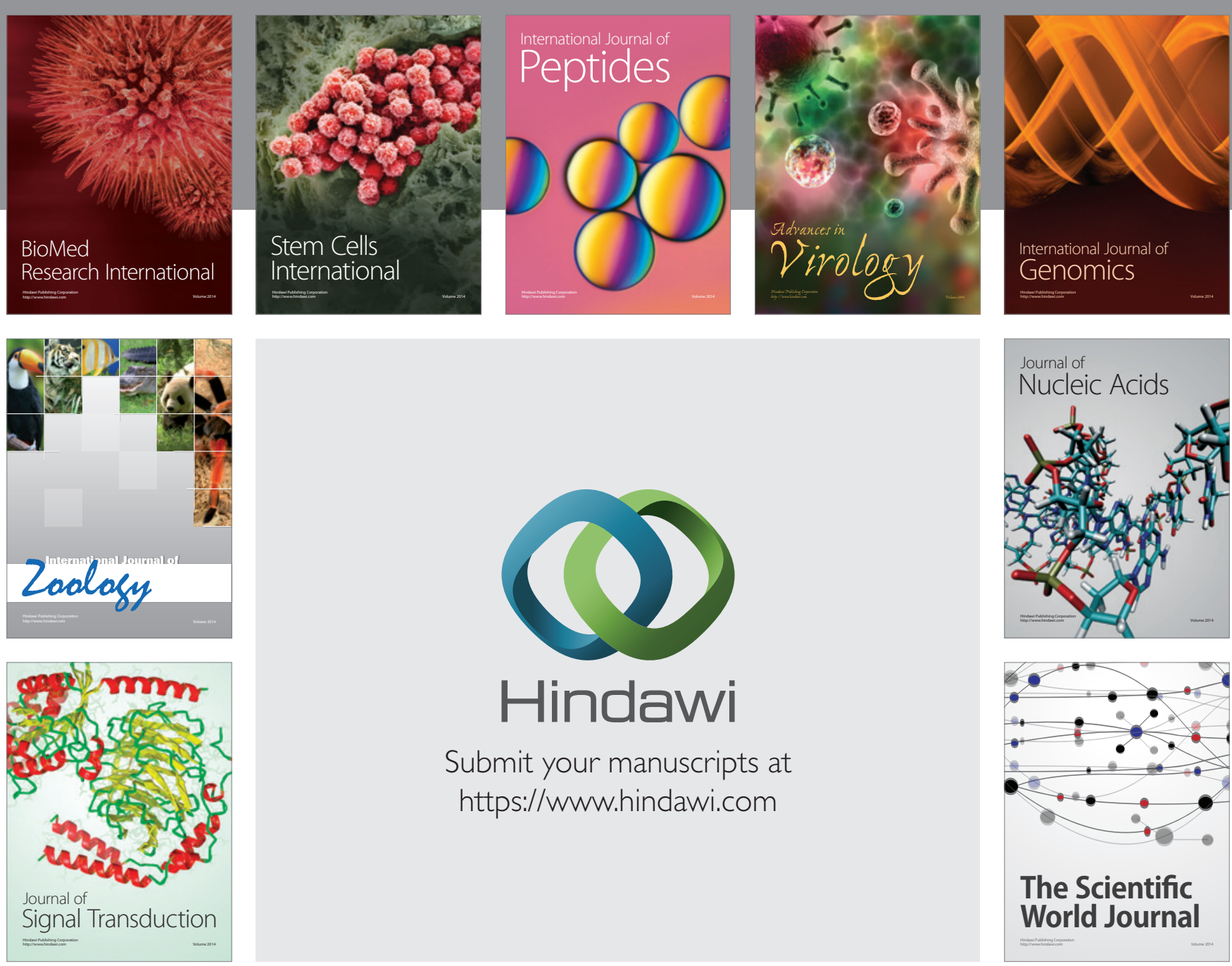

Submit your manuscripts at

https://www.hindawi.com
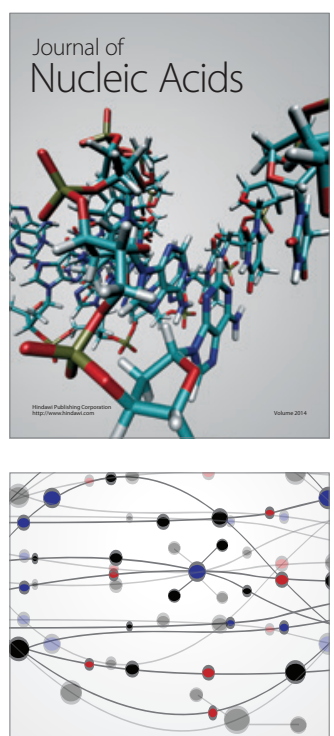

The Scientific World Journal
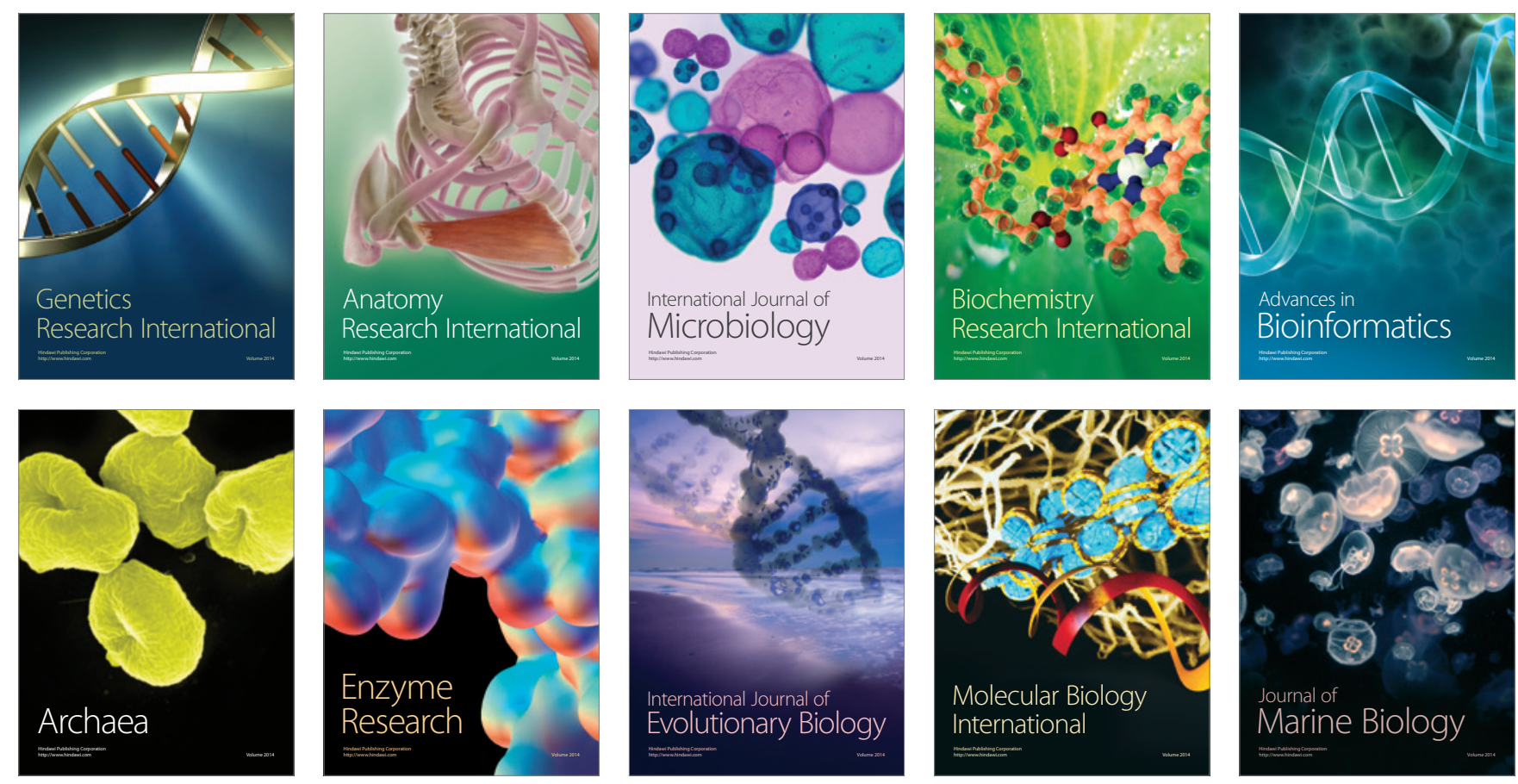